\title{
Positive quandle homology and its applications in knot theory
}

\author{
ZHIYUN CHENG \\ HONGZHU GAO
}

\begin{abstract}
Algebraic homology and cohomology theories for quandles have been studied extensively in recent years. With a given quandle 2 -cocycle (3-cocycle) one can define a state-sum invariant for knotted curves (surfaces). In this paper we introduce another version of quandle (co)homology theory, called positive quandle (co)homology. Some properties of positive quandle (co)homology groups are given and some applications of positive quandle cohomology in knot theory are discussed.
\end{abstract}

57M25, 57M27; 57Q45

\section{Introduction}

In knot theory, by considering representations from the knot group onto the dihedral group of order $2 n$ one obtains a family of elementary knot invariants, known as Fox $n$-colorings; see Fox [14]. Quandles, sets with certain self-distributive operations satisfying axioms analogous to the Reidemeister moves, were first proposed by D Joyce [20] and S V Matveev [25] independently. For a given quandle $X$, one can define a quandle coloring invariant by counting the quandle homomorphisms from the fundamental quandle of a knot to $X$. For the fundamental quandle and its presentations the reader is referred to [20] and Fenn and Rourke [11]. Equivalently speaking, one can label each arc of a knot diagram by an element of a fixed quandle, subject to certain constraints. The quandle coloring invariant can be computed by counting the number of these labellings. It is natural to consider how to improve this integer-valued knot invariant. Since the quandle coloring invariant equals the number of different proper colorings, it is natural to associate a weight function to each colored knot diagram which does not depend on the choice of the knot diagram. In this way, instead of several colored knot diagrams one will obtain several weight functions and the number of these weight functions is exactly the quandle coloring invariant. Carter, Jelsovsky, Kamada, Langford and Saito [3] associated a Boltzmann weight to each crossing and then considered the signed product of Boltzmann weights for all crossing points. In fact, based on R Fenn, C Rourke and B Sanderson's framework of rack and quandle homology [12; 13], Carter 
et al described a homology theory for quandles such that each 2-cocycle and 3-cocycle can be used to define a state-sum invariant for knots and knotted surfaces respectively. Many applications of quandle cocycle invariants have been investigated in the past decade. For example, with a suitable choice of 3-cocycle from the dihedral quandle $R_{3}$, one can prove the chirality of the trefoil; see Kamada [21]. For knotted surfaces, by using cocycle invariants it was proved that the 2 -twist spun trefoil is noninvertible and has triple point number 4; see [3] and Satoh and Shima [35].

In this paper we introduce another quandle homology and cohomology theory, called positive quandle homology and positive quandle cohomology. The definition of positive quandle (co)homology is similar to that of the original quandle (co)homology. It is not surprising that positive quandle homology shares many common properties with quandle homology, which will be discussed in Section 4. The most interesting part of this new quandle (co)homology theory is that it also can be used to define cocycle invariants for knots and knotted surfaces. Some properties of quandle homology and quandle cocycle invariants have their corresponding versions in positive quandle homology theory. This phenomenon suggests that quandle homology theory and positive quandle homology theory are parallel to each other, and in some special cases (Proposition 3.3) they coincide with each other. However the positive quandle cocycle invariants reflect quite different information compared with that of the quandle cocycle invariants. In a sense, the quandle cocycle invariants concern the signed crossings of a knot diagram but the positive quandle cocycle invariants concern the alternating information of a knot diagram. Our hope is that these new knot invariants can offer some hints to the study of crossing numbers via quandle homology theory.

The rest of this paper is arranged as follows. In Section 2, a brief review of quandle structure and quandle coloring invariant is given. Some applications of quandle coloring invariants in knot theory will also be discussed. In Section 3, we give the definition of positive quandle homology and cohomology. The relation between positive quandle (co)homology and quandle (co)homology will also be studied. Section 4 is devoted to the calculation of positive quandle homology and cohomology. We will calculate the positive quandle homology for some simple quandles. In Section 5, we show how to use positive quandle 2-cocycle and 3-cocycle to define invariants for knots and knotted surfaces respectively. We end this paper with two examples which study the trivially colored crossing points of a knot diagram, from which the motivation of this study arises.

\section{Quandle and quandle coloring invariants}

First we give a short review of the definition of quandle. 
Definition 2.1 A quandle $(X, *)$, is a set $X$ with a binary operation $(a, b) \rightarrow a * b$ satisfying the following axioms:

(1) For any $a \in X, a * a=a$.

(2) For any $b, c \in X$, there exists a unique $a \in X$ such that $a * b=c$.

(3) For any $a, b, c \in X,(a * b) * c=(a * c) *(b * c)$.

Usually we simply denote a quandle $(X, *)$ by $X$. If a nonempty set $X$ with a binary operation $(a, b) \rightarrow a * b$ satisfies the second and the third axioms, then we call it a rack. In particular if a quandle $X$ satisfies a modified version of the second axiom "for any $b, c \in X,(c * b) * b=c$," ie the unique element $a=c * b$, we call such a quandle an involutory quandle [20] or kei; see Takasaki [36]. The relation below follows directly from the definitions above:

$$
\{\text { keis }\} \subset\{\text { quandles }\} \subset\{\text { racks }\} .
$$

In the second axiom we usually denote the element $a$ by $a=c *^{-1} b$. It is not difficult to observe that $\left(X, *^{-1}\right)$ also defines a quandle structure, which is usually called the dual quandle of $(X, *)$. We denote the dual of $X$ by $X^{*}$. Note that a quandle is an involutory quandle if and only if $*=*^{-1}$.

Next we list some common examples of quandles (see [11; 20], Ho and Nelson [17] and Vendramin [37] for more examples):

- The trivial quandle of order $n: T_{n}=\left\{a_{1}, \ldots, a_{n}\right\}$ with $a_{i} * a_{j}=a_{i}$.

- The dihedral quandle of order $n: R_{n}=\{0, \ldots, n-1\}$ with $i * j=2 j-i(\bmod n)$.

- The conjugation quandle: a conjugacy class $X$ of a group $G$ with $a * b=b^{-1} a b$.

- The Alexander quandle: a $Z\left[t, t^{-1}\right]$-module $M$ with $a * b=t a+(1-t) b$.

From now on all the quandles mentioned throughout are assumed to be finite quandles. To a given finite quandle $X$ we can define an associated integer-valued knot invariant $\mathrm{Col}_{X}(K)$, the quandle coloring invariant. Let $K$ be a knot diagram. We will often abuse our notation, letting $K$ refer both to a knot diagram and the knot itself. It is not difficult to determine the meaning that is intended from the context. A coloring of $K$ by a given quandle $X$ is a map from the set of arcs of $K$ to the elements of $X$. We say a coloring is proper if at each crossing the images of the map satisfy the relation given in Figure 1.

Now we define the quandle coloring invariant $\operatorname{Col}_{X}(K)$ to be the number of proper colorings of $K$ by the quandle $X$. Since $X$ is finite, this definition makes sense. It is well known that although the definition of $\operatorname{Col}_{X}(K)$ depends on the choice of a 


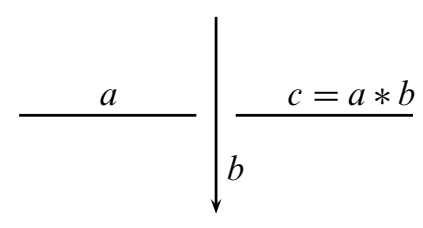

Figure 1: The proper coloring rule

knot diagram, however the integer $\operatorname{Col}_{X}(K)$ is independent of the knot diagram. In fact the three axioms from the definition of quandle structure correspond to the three Reidemeister moves. In particular, $\operatorname{Col}_{X}(K) \geq n$ if $X$ contains $n$ elements, since there always exist $n$ trivial colorings. When $X=R_{n}$, we have $\operatorname{Col}_{R_{n}}(K)=\operatorname{Col}_{n}(K)$, the number of distinct Fox $n$-colorings of $K$ [14]. It is well known that $\operatorname{Col}_{n}(K)$ equals the number of distinct representations from the knot group $\pi_{1}\left(R^{3} \backslash K\right)$ to the dihedral group of order $2 n$. As a generalization of Fox $n$-coloring, $\operatorname{Col}_{X}(K)$ is equivalent to the number of quandle homomorphisms from the fundamental quandle of $K$ to $X$. Here the fundamental quandle of $K$ is defined by assigning generators to arcs, and certain relations to crossings, which is quite similar to the presentation of the knot group. See [20; 25] for more details.

Before ending this section we list some properties of the quandle coloring invariant.

- We have $\operatorname{Col}_{X}(K)=\operatorname{Col}_{X}\left(\bar{K}^{*}\right)$. Here $\bar{K}^{*}$ denotes the mirror image of $K$ with the reversed orientation. This follows from the fact that the fundamental quandles of $K$ and $\bar{K}^{*}$ are isomorphic [20;25].

- We have $\log _{|X|}\left(\operatorname{Col}_{X}(K)\right) \leq b(K)$ and $\log _{|X|}\left(\operatorname{Col}_{X}(K)\right) \leq u(K)+1$; see Przytycki [29]. Here $|X|$ denotes the order of $X, b(K)$ and $u(K)$ denote the bridge number and unknotting number respectively. The reader is referred to Clark, Elhamdadi, Saito and Yeatman [9] for some recent progress on the applications of quandle coloring invariants.

- $\operatorname{Col}_{X}(K)$ is not a Vassiliev invariant if $\operatorname{Col}_{X}(K)$ is not constant. This can be proved with the similar idea of [10], in which M Eisermann proved that $\operatorname{Col}_{n}(K)$ is not a Vassiliev invariant. Briefly speaking, in [10] it was proved that if a Vassiliev invariant $F$ is bounded on any given vertical twist sequence, then $F$ is constant. On the other hand, for any fixed vertical twist sequence the braid index is bounded by some integer, say $b$. It is not difficult to show that the fundamental quandle of each knot of this vertical twist sequence can be generated by at most $b$ elements. Assume $X$ contains $n$ elements. Then we deduce that $\operatorname{Col}_{X}(K) \leq n^{b}$. Because $\operatorname{Col}_{X}(K)$ is not constant, $\operatorname{Col}_{X}(K)$ is not a Vassiliev invariant. 


\section{Homology and cohomology theory for quandles}

Rack (co)homology theory was first defined by Fenn, Rourke and Sanderson in [13]. It is similar to group (co)homology theory. Quandle (co)homology was proposed by Carter, Jelsovsky, Kamada, Langford and Saito [3] as a modification of the rack (co)homology. As an application, they defined state-sum invariants for knots and knotted surfaces by using quandle cocycles. Some calculations of quandle homology groups and the associated state-sum invariants can be found in Carter, Jelsovsky, Kamada and Saito [4; 5], Mochizuki [26] and Niebrzydowski and Przytycki [27], or see Carter [1] for a good survey. Recently, the (co)homology theory of sets with distributive operations was systematically studied by Przytycki; the readers are referred to Przytycki [30] and Przytycki and Sikora [31] for more details. We give a short review of the construction of the quandle (co)homology group, then we will give the definition of positive quandle (co)homology group.

Assume $X$ is a finite quandle. Let $C_{n}^{R}(X)$ denote the free abelian group generated by $n-$ tuples $\left(a_{1}, \ldots, a_{n}\right)$, where $a_{i} \in X$. In order to make $C_{n}^{R}(X)$ into a chain complex, let us consider the following two homomorphisms from $C_{n}^{R}(X)$ to $C_{n-1}^{R}(X)$; here $\bar{a}_{i}$ denotes the omission of the element $a_{i}$ :

$$
\begin{aligned}
& d_{1}\left(a_{1}, \ldots, a_{n}\right)=\sum_{i=1}^{n}(-1)^{i}\left(a_{1}, \ldots, \bar{a}_{i}, \ldots, a_{n}\right) \quad(n \geq 2), \\
& d_{2}\left(a_{1}, \ldots, a_{n}\right)=\sum_{i=1}^{n}(-1)^{i}\left(a_{1} * a_{i}, \ldots, a_{i-1} * a_{i}, a_{i+1}, \ldots, a_{n}\right) \quad(n \geq 2), \\
& d_{i}\left(a_{1}, \ldots, a_{n}\right)=0 \quad(n \leq 1, i=1,2) .
\end{aligned}
$$

For the two homomorphisms $d_{1}, d_{2}$ defined above, we have the following lemma.

Lemma 3.1 We have $d_{1}^{2}=d_{2}^{2}=d_{1} d_{2}+d_{2} d_{1}=0$.

Proof One computes

$$
\begin{aligned}
d_{1}^{2}\left(a_{1}, \ldots, a_{n}\right) & =d_{1}\left(\sum_{i=1}^{n}(-1)^{i}\left(a_{1}, \ldots, \bar{a}_{i}, \ldots, a_{n}\right)\right) \\
& =\sum_{i=1}^{n}(-1)^{i}\left(\sum_{j<i}(-1)^{j}\left(a_{1}, \ldots, \bar{a}_{j}, \ldots, \bar{a}_{i}, \ldots, a_{n}\right)\right. \\
& \left.\quad+\sum_{j>i}(-1)^{j-1}\left(a_{1}, \ldots, \bar{a}_{i}, \ldots, \bar{a}_{j}, \ldots, a_{n}\right)\right) \\
= & 0,
\end{aligned}
$$




$$
\begin{aligned}
& d_{2}^{2}\left(a_{1}, \ldots, a_{n}\right) \\
& =d_{2}\left(\sum_{i=1}^{n}(-1)^{i}\left(a_{1} * a_{i}, \ldots, a_{i-1} * a_{i}, a_{i+1}, \ldots, a_{n}\right)\right) \\
& =\sum_{i=1}^{n}(-1)^{i}\left(\sum _ { j < i } ( - 1 ) ^ { j } \left(\left(a_{1} * a_{i}\right) *\left(a_{j} * a_{i}\right), \ldots,\left(a_{j-1} * a_{i}\right) *\left(a_{j} * a_{i}\right), a_{j+1} * a_{i}, \ldots,\right.\right. \\
& \quad+\sum_{j>i}(-1)^{j-1}\left(\left(a_{1} * a_{i}\right) * a_{j}, \ldots, a_{i}, a_{i+1}, \ldots, a_{n}\right) \\
& \quad \begin{array}{r}
\left.\left.a_{j-1} * a_{j}, a_{j+1}, \ldots, a_{n}\right)\right) \\
=0,
\end{array}
\end{aligned}
$$

$$
d_{1} d_{2}\left(a_{1}, \ldots, a_{n}\right)+d_{2} d_{1}\left(a_{1}, \ldots, a_{n}\right)
$$

$$
\begin{aligned}
& =d_{1}\left(\sum_{i=1}^{n}(-1)^{i}\left(a_{1} * a_{i}, \ldots, a_{i-1} * a_{i}, a_{i+1}, \ldots, a_{n}\right)\right) \\
& \quad+d_{2}\left(\sum_{i=1}^{n}(-1)^{i}\left(a_{1}, \ldots, \bar{a}_{i}, \ldots, a_{n}\right)\right) \\
& =\sum_{i=1}^{n} \sum_{j<i}(-1)^{i+j}\left(a_{1} * a_{i}, \ldots, \overline{a_{j} * a_{i}}, \ldots, a_{i-1} * a_{i}, a_{i+1}, \ldots, a_{n}\right)
\end{aligned}
$$$$
+\sum_{i=1}^{n} \sum_{j>i}(-1)^{i+j-1}\left(a_{1} * a_{i}, \ldots, a_{i-1} * a_{i}, a_{i+1}, \ldots, \bar{a}_{j}, \ldots, a_{n}\right)
$$$$
+\sum_{i=1}^{n} \sum_{j<i}(-1)^{i+j}\left(a_{1} * a_{j}, \ldots, a_{j-1} * a_{j}, a_{j+1}, \ldots, \bar{a}_{i}, \ldots, a_{n}\right)
$$$$
+\sum_{i=1}^{n} \sum_{j>i}(-1)^{i+j-1}\left(a_{1} * a_{j}, \ldots, \overline{a_{i} * a_{j}}, \ldots, a_{j-1} * a_{j}, a_{j+1}, \ldots, a_{n}\right)
$$

$=0$,

thus completing the proof.

Lemma 3.1 suggests we should investigate the four chain complexes $\left\{C_{n}^{R}(X), d_{1}\right\}$, $\left\{C_{n}^{R}(X), d_{2}\right\},\left\{C_{n}^{R}(X), d_{1}+d_{2}\right\}$ and $\left\{C_{n}^{R}(X), d_{1}-d_{2}\right\}$. Note that $\left\{C_{n}^{R}(X), d_{1}\right\}$ is acyclic. A Inoue and Y Kabaya [19] regarded $\left\{C_{n}^{R}(X), d_{1}\right\}$ as a right $\mathbb{Z}\left[G_{X}\right]-$ module, where $G_{X}$ denotes the associated group of $X$, ie $G_{X}$ is generated by the elements of $X$ and satisfies the relation $a * b=b^{-1} a b$. With this viewpoint they defined the simplicial quandle homology to be the homology group of the chain complex $\left\{C_{n}^{R}(X) \otimes_{\mathbb{Z}\left[G_{X}\right]}\right.$ $\left.\mathbb{Z}, d_{1}\right\}$; see [19] for more details. On the other hand, we remark that $\left\{C_{n}^{R}(X), d_{2}\right\}$ is 
also acyclic [31]. In fact let us consider the map $f(x)=x * x_{0}: X \rightarrow X$, where $x_{0}$ is a fixed element of $X$. Then $f$ induces a chain map $C_{*}^{R}(X) \rightarrow C_{*}^{R}(X)$ which is chain homotopic to the zero map by the homotopy map $\left(x_{1}, \ldots, x_{n}\right) \rightarrow\left(x_{1}, \ldots, x_{n}, x_{0}\right)$. It follows that $\mathrm{id}=f_{*}\left(f^{-1}\right)_{*}=0$, then one concludes that $\left\{C_{n}^{R}(X), d_{2}\right\}$ is acyclic.

Assume $X$ is a fixed finite quandle. Let $C_{n}^{D}(X)(n \geq 2)$ denote the free abelian group generated by $n$-tuples $\left(a_{1}, \ldots, a_{n}\right)$ with $a_{i}=a_{i+1}$ for some $1 \leq i \leq n-1$, and $C_{n}^{D}(X)=0$ if $n \leq 1$. The following lemma tells us that $\left\{C_{n}^{D}(X), d_{1} \pm d_{2}\right\}$ is a subcomplex of $\left\{C_{n}^{R}(X), d_{1} \pm d_{2}\right\}$.

Lemma 3.2 We have that $\left\{C_{n}^{D}(X), d_{i}\right\}$ is a subcomplex of $\left\{C_{n}^{R}(X), d_{i}\right\}(i=1,2)$.

Proof Choose an $n$-tuple $\left(a_{1}, \ldots, a_{i}, a_{i+1}, \ldots, a_{n}\right) \in C_{n}^{D}(X)$, where $a_{i}=a_{i+1}$. One computes

$$
\begin{aligned}
& d_{1}\left(a_{1}, \ldots, a_{i}, a_{i+1}, \ldots, a_{n}\right) \\
& =\sum_{j<i}(-1)^{j}\left(a_{1}, \ldots, \bar{a}_{j}, \ldots, a_{i}, a_{i+1}, \ldots, a_{n}\right) \\
& \quad+\sum_{j>i+1}(-1)^{j}\left(a_{1}, \ldots, a_{i}, a_{i+1}, \ldots, \bar{a}_{j}, \ldots, a_{n}\right) \\
& +(-1)^{i}\left(a_{1}, \ldots, \bar{a}_{i}, a_{i+1}, \ldots, a_{n}\right)+(-1)^{i+1}\left(a_{1}, \ldots, a_{i}, \bar{a}_{i+1}, \ldots, a_{n}\right) \\
& =\sum_{j<i}(-1)^{j}\left(a_{1}, \ldots, \bar{a}_{j}, \ldots, a_{i}, a_{i+1}, \ldots, a_{n}\right) \\
& \quad+\sum_{j>i+1}(-1)^{j}\left(a_{1}, \ldots, a_{i}, a_{i+1}, \ldots, \bar{a}_{j}, \ldots, a_{n}\right) \in C_{n-1}^{D}(X),
\end{aligned}
$$

$d_{2}\left(a_{1}, \ldots, a_{i}, a_{i+1}, \ldots, a_{n}\right)$

$$
\begin{aligned}
& =\sum_{j<i}(-1)^{j}\left(a_{1} * a_{j}, \ldots, a_{j-1} * a_{j}, a_{j+1}, \ldots, a_{i}, a_{i+1}, \ldots, a_{n}\right) \\
& \quad+\sum_{j>i+1}(-1)^{j}\left(a_{1} * a_{j}, \ldots, a_{i} * a_{j}, a_{i+1} * a_{j}, \ldots, a_{j-1} * a_{j}, a_{j+1}, \ldots, a_{n}\right) \\
& \quad+(-1)^{i}\left(a_{1} * a_{i}, \ldots, a_{i-1} * a_{i}, a_{i+1}, \ldots, a_{n}\right) \\
& \quad+(-1)^{i+1}\left(a_{1} * a_{i+1}, \ldots, a_{i} * a_{i+1}, a_{i+2}, \ldots, a_{n}\right) \\
& =\sum_{j<i}(-1)^{j}\left(a_{1} * a_{j}, \ldots, a_{j-1} * a_{j}, a_{j+1}, \ldots, a_{i}, a_{i+1}, \ldots, a_{n}\right) \\
& \quad+\sum_{j>i+1}(-1)^{j}\left(a_{1} * a_{j}, \ldots, a_{i} * a_{j}, a_{i+1} * a_{j}, \ldots, a_{j-1} * a_{j}, a_{j+1}, \ldots, a_{n}\right) \\
& \in C_{n-1}^{D}(X),
\end{aligned}
$$

thus completing the proof.

Defining $C_{n}^{Q}(X)=C_{n}^{R}(X) / C_{n}^{D}(X)$, we have two chain complexes $\left\{C_{n}^{Q}(X), d_{1} \pm d_{2}\right\}$, where $d_{1} \pm d_{2}$ denotes the induced homomorphisms. For simplicity, we use $\partial^{+}$and $\partial^{-}$ 
to denote $d_{1}+d_{2}$ and $d_{1}-d_{2}$ respectively, and use $C_{*}^{W \pm}(X)$ to denote $\left\{C_{*}^{W}(X), \partial_{*}^{ \pm}\right\}$ $(W \in\{R, D, Q\})$. For an abelian group $G$, define the the chain complex $C_{*}^{W \pm}(X ; G)$ and cochain complex $C_{W \pm}^{*}(X ; G)$ as below $(W \in\{R, D, Q\})$ :

- $C_{*}^{W \pm}(X ; G)=C_{*}^{W \pm}(X) \otimes G, \partial_{*}^{ \pm}=\partial_{*}^{ \pm} \otimes \mathrm{id}$.

- $C_{W \pm}^{*}(X ; G)=\operatorname{Hom}\left(C_{*}^{W \pm}(X), G\right), \delta_{ \pm}^{*}=\operatorname{Hom}\left(\partial_{*}^{ \pm}, \mathrm{id}\right)$.

The positive quandle (co)homology groups of $X$ with coefficient $G$ are defined to be the (co)homology groups of the (co)chain complex $C_{*}^{Q+}(X ; G)\left(C_{Q+}^{*}(X ; G)\right)$, and the negative quandle (co)homology groups of a quandle $X$ with coefficient $G$ are defined to be the (co)homology groups of the (co)chain complex $C_{*}^{Q-}(X ; G)$ $\left(C_{Q-}^{*}(X ; G)\right)$. In other words,

$$
H_{n}^{Q \pm}(X ; G)=H_{n}\left(C_{*}^{Q \pm}(X ; G)\right) \quad \text { and } \quad H_{Q \pm}^{n}(X ; G)=H^{n}\left(C_{Q \pm}^{*}(X ; G)\right) .
$$

Similarly we define the \pm rack (co)homology groups and \pm degeneration (co)homology groups by

$$
\begin{array}{lll}
H_{n}^{R \pm}(X ; G)=H_{n}\left(C_{*}^{R \pm}(X ; G)\right) & \text { and } & H_{R \pm}^{n}(X ; G)=H^{n}\left(C_{R \pm}^{*}(X ; G)\right), \\
H_{n}^{D \pm}(X ; G)=H_{n}\left(C_{*}^{D \pm}(X ; G)\right) & \text { and } \quad H_{D \pm}^{n}(X ; G)=H^{n}\left(C_{D \pm}^{*}(X ; G)\right) .
\end{array}
$$

The reader has recognized that the negative quandle (co)homology groups are nothing but the quandle (co)homology groups introduced by Carter et al in [3]. Therefore we will still use the name quandle (co)homology instead of nagative quandle (co)homology,

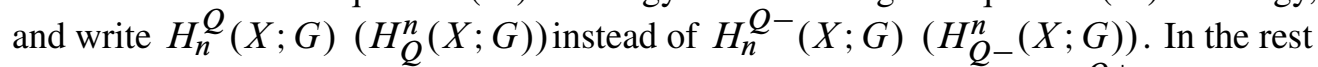
of this paper we will focus on the positive quandle homology groups $H_{*}^{Q+}(X ; G)$ and cohomology groups $H_{Q+}^{*}(X ; G)$. In particular, when $G=\mathbb{Z}_{2}$, the following result is obvious.

Proposition 3.3 We have that

$$
H_{n}^{Q+}\left(X ; \mathbb{Z}_{2}\right) \cong H_{n}^{Q}\left(X ; \mathbb{Z}_{2}\right) \text { and } H_{Q+}^{n}\left(X ; \mathbb{Z}_{2}\right) \cong H_{Q}^{n}\left(X ; \mathbb{Z}_{2}\right) \text {. }
$$

We end this section by listing the positive quandle 2-cocycle condition and positive quandle 3-cocycle condition. Later it will be shown that they are related to the third Reidemeister move of knots and the tetrahedral move of knotted surfaces. The readers are suggested to compare these with the quandle 2-cocycle condition and quandle 3 -cocycle condition given in [3].

- A positive quandle 2-cocycle $\phi$ satisfies the condition

$$
-\phi(b, c)-\phi(b, c)+\phi(a, c)+\phi(a * b, c)-\phi(a, b)-\phi(a * c, b * c)=0 .
$$


- A positive quandle 3 -cocycle $\theta$ satisfies the condition

$$
\begin{aligned}
& -\theta(b, c, d)-\theta(b, c, d)+\theta(a, c, d)+\theta(a * b, c, d) \\
& \quad-\theta(a, b, d)-\theta(a * c, b * c, d)+\theta(a, b, c)+\theta(a * d, b * d, c * d)=0 .
\end{aligned}
$$

\section{Computing positive quandle homology and cohomology}

This section is devoted to the calculation of positive quandle homology and cohomology for some simple examples. Before this, we need to discuss some basic properties of the positive quandle homology and cohomology. Most of these results have their corresponding versions in quandle homology theory.

First it was pointed out that since $\left\{C_{n}^{Q}(X)\right\}$ is a chain complex of free abelian groups, there is a universal coefficient theorem for quandle homology and quandle cohomology [5]. For the same reason, there also exists a universal coefficient theorem for positive quandle homology and cohomology.

Theorem 4.1 (Universal coefficient theorem) For a given quandle $X$, there is a pair of split exact sequences

$$
\begin{gathered}
0 \rightarrow H_{n}^{Q+}(X ; \mathbb{Z}) \otimes G \rightarrow H_{n}^{Q+}(X ; G) \rightarrow \operatorname{Tor}\left(H_{n-1}^{Q+}(X ; \mathbb{Z}), G\right) \rightarrow 0, \\
0 \rightarrow \operatorname{Ext}\left(H_{n-1}^{Q+}(X ; \mathbb{Z}), G\right) \rightarrow H_{Q+}^{n}(X ; G) \rightarrow \operatorname{Hom}\left(H_{n}^{Q+}(X ; \mathbb{Z}), G\right) \rightarrow 0 .
\end{gathered}
$$

The universal coefficient theorem tells us that it suffices to study the positive quandle homology and cohomology groups with integer coefficients. As usual we will omit the coefficient group $G$ if $G=\mathbb{Z}$. The following lemma gives an example of the computation of the simplest nontrivial quandle $R_{3}$ in detail.

Lemma 4.2 We have $H_{Q+}^{2}\left(R_{3}\right) \cong \mathbb{Z}_{3}$.

Proof Recall we have that $R_{3}=\{0,1,2\}$ with quandle operations $i * j=2 j-$ $i(\bmod 3)$. Choose a positive quandle 2-cocycle $\phi \in Z_{Q+}^{2}\left(R_{3}\right)$. We assume that $\phi=\sum_{i, j \in\{0,1,2\}} c_{(i, j)} \chi_{(i, j)}$, where $\chi_{(i, j)}$ denotes the characteristic function

$$
\chi_{(i, j)}(k, l)= \begin{cases}1 & \text { if }(i, j)=(k, l), \\ 0 & \text { if }(i, j) \neq(k, l) .\end{cases}
$$

Recall that $\phi(i, i)=0$, ie $c_{(i, i)}=0$.

Next we need to investigate the positive quandle 2-cocycle conditions

$$
-\phi(j, k)-\phi(j, k)+\phi(i, k)+\phi(i * j, k)-\phi(i, j)-\phi(i * k, j * k)=0
$$


for all triples $(i, j, k)$ from $\{0,1,2\}$. There are 12 equations in total on $c_{(i, j)}$ :

$$
\begin{aligned}
& -2 c_{(1,0)}+c_{(2,0)}-c_{(0,1)}-c_{(0,2)}=0, \quad-2 c_{(2,0)}+c_{(1,0)}-c_{(0,2)}-c_{(0,1)}=0, \\
& -2 c_{(0,1)}+c_{(2,1)}-c_{(1,0)}-c_{(1,2)}=0, \quad-2 c_{(2,1)}+c_{(0,1)}-c_{(1,2)}-c_{(1,0)}=0, \\
& -2 c_{(0,2)}+c_{(1,2)}-c_{(2,0)}-c_{(2,1)}=0, \quad-2 c_{(1,2)}+c_{(0,2)}-c_{(2,1)}-c_{(2,0)}=0, \\
& -2 c_{(1,2)}+c_{(0,2)}-c_{(0,1)}-c_{(1,0)}=0, \quad-2 c_{(2,1)}+c_{(0,1)}-c_{(0,2)}-c_{(2,0)}=0 \text {, } \\
& -2 c_{(0,2)}+c_{(1,2)}-c_{(1,0)}-c_{(0,1)}=0, \quad-2 c_{(2,0)}+c_{(1,0)}-c_{(1,2)}-c_{(2,1)}=0 \text {, } \\
& -2 c_{(0,1)}+c_{(2,1)}-c_{(2,0)}-c_{(0,2)}=0, \quad-2 c_{(1,0)}+c_{(2,0)}-c_{(2,1)}-c_{(1,2)}=0 .
\end{aligned}
$$

After simplifying the equations above we obtain

$$
\begin{array}{lll}
c_{(0,1)}=z, & c_{(1,0)}=-y-z, & c_{(0,2)}=y, \\
c_{(2,0)}=-y-z, & c_{(1,2)}=y, & c_{(2,1)}=z .
\end{array}
$$

Here we put $c_{(1,2)}=y$ and $c_{(2,1)}=z$. Hence we have the following equation for the positive quandle 2-cocycle:

$$
\phi=y\left(\chi_{(0,2)}+\chi_{(1,2)}-\chi_{(1,0)}-\chi_{(2,0)}\right)+z\left(\chi_{(0,1)}+\chi_{(2,1)}-\chi_{(1,0)}-\chi_{(2,0)}\right) .
$$

On the other hand, we have

$$
\begin{aligned}
& \delta \chi_{0}=\left(\chi_{(0,2)}+\chi_{(1,2)}-\chi_{(1,0)}-\chi_{(2,0)}\right)+\left(\chi_{(0,1)}+\chi_{(2,1)}-\chi_{(1,0)}-\chi_{(2,0)}\right), \\
& \delta \chi_{1}=\left(\chi_{(1,0)}+\chi_{(2,0)}-\chi_{(0,1)}-\chi_{(2,1)}\right)+\left(\chi_{(0,2)}+\chi_{(1,2)}-\chi_{(0,1)}-\chi_{(2,1)}\right), \\
& \delta \chi_{2}=\left(\chi_{(0,1)}+\chi_{(2,1)}-\chi_{(0,2)}-\chi_{(1,2)}\right)+\left(\chi_{(1,0)}+\chi_{(2,0)}-\chi_{(0,2)}-\chi_{(1,2)}\right) .
\end{aligned}
$$

Since

$$
\phi=y\left(\delta \chi_{0}\right)+(z-y)\left(\chi_{(0,1)}+\chi_{(2,1)}-\chi_{(1,0)}-\chi_{(2,0)}\right),
$$

then

$$
H_{Q+}^{2}\left(R_{3}\right) \cong\left\{\chi_{(0,1)}+\chi_{(2,1)}-\chi_{(1,0)}-\chi_{(2,0)} \mid \delta \chi_{0}, \delta \chi_{1}\right\} .
$$

From $\delta \chi_{0}=\delta \chi_{1}=0$ one can easily deduce that $3\left(\chi_{(0,1)}+\chi_{(2,1)}-\chi_{(1,0)}-\chi_{(2,0)}\right)=0$. It follows that $H_{Q+}^{2}\left(R_{3}\right) \cong \mathbb{Z}_{3}$.

Note that the second quandle cohomology group of $R_{3}$ is trivial, $H_{Q}^{2}\left(R_{3} ; \mathbb{Z}\right) \cong 0$ [3]. According to the definition $C_{n}^{Q}(X)=C_{n}^{R}(X) / C_{n}^{D}(X)$, there is a short exact sequence

$$
0 \rightarrow C_{*}^{D}(X) \rightarrow C_{*}^{R}(X) \rightarrow C_{*}^{Q}(X) \rightarrow 0
$$

of chain complexes. It follows that there is a long exact sequence of homology groups

$$
\cdots \rightarrow H_{n}^{D}(X) \rightarrow H_{n}^{R}(X) \rightarrow H_{n}^{Q}(X) \rightarrow H_{n-1}^{D}(X) \rightarrow \cdots
$$


In [5], it was conjectured that the short exact sequence of chain complexes above is split. Later R A Litherland and S Nelson gave an affirmative answer to this conjecture in [23]. The following theorem says that the splitting map defined by R A Litherland and S Nelson still works in positive quandle homology theory.

Theorem 4.3 For a given quandle $X$, there exists a short exact sequence

$$
0 \rightarrow H_{n}^{D+}(X) \rightarrow H_{n}^{R+}(X) \rightarrow H_{n}^{Q+}(X) \rightarrow 0 .
$$

Proof According to the definition of positive homology groups there exists a short exact sequence

$$
0 \rightarrow C_{*}^{D+}(X) \stackrel{u_{*}}{\longrightarrow} C_{*}^{R+}(X) \stackrel{v_{*}}{\longrightarrow} C_{*}^{Q+}(X) \rightarrow 0 .
$$

It suffices to find a chain map $w_{n}: C_{n}^{R+}(X) \rightarrow C_{n}^{D+}(X)$ such that $w_{n} \circ u_{n}=\mathrm{id}$. We use the splitting map $w_{n}(c)=c-\alpha_{n}(c)$ introduced by R A Litherland and S Nelson in [23]; here $c \in C_{n}^{R+}(X)$, and $\alpha_{n}$ is defined by $\alpha_{n}\left(a_{1}, \ldots, a_{n}\right)=\left(a_{1}, a_{2}-a_{1}, \ldots, a_{n}-a_{n-1}\right)$ on $n$-tuples and extending linearly to $C_{n}^{R+}(X)$. The following two relationships will be frequently used during the proof; note that the notation we use here is a bit different from that in [23].

- We have

$$
\begin{aligned}
\partial^{+}\left(a_{1}, \ldots, a_{n+1}\right)=\left(\partial^{+}\left(a_{1}, \ldots, a_{n}\right), a_{n+1}\right) \\
+(-1)^{n+1}\left(\left(a_{1}, \ldots, a_{n}\right)+\left(a_{1}, \ldots, a_{n}\right) * a_{n+1}\right)
\end{aligned}
$$

here the notation $\left(a_{1}, \ldots, a_{n}\right) * a_{n+1}$ denotes $\left(a_{1} * a_{n+1}, \ldots, a_{n} * a_{n+1}\right)$.

- We have $\alpha_{n+1}\left(a_{1}, \ldots, a_{n+1}\right)=\left(\alpha_{n}\left(a_{1}, \ldots, a_{n}\right), a_{n+1}\right)-\left(\alpha_{n}\left(a_{1}, \ldots, a_{n}\right), a_{n}\right)$. Generally, we write

$$
\alpha_{n+1}\left(c, a_{n+1}\right)=\left(\alpha_{n}(c), a_{n+1}\right)-\left(\alpha_{n}(c), l(c)\right) ;
$$

here $c \in C_{n}^{R+}(X)$ and $l(c) \in C_{1}^{R+}(X)$. In particular $l\left(a_{1}, \ldots, a_{n}\right)=a_{n}$.

First we show that $c-\alpha_{n}(c) \in C_{n}^{D+}(X)$ and $w_{n} \circ u_{n}=\mathrm{id}$. In order to prove $c-\alpha_{n}(c) \in$ $C_{n}^{D+}(X)$ it is sufficient to consider the case $c=\left(a_{1}, \ldots, a_{n}\right) \in C_{n}^{R+}(X)$. Note that

$$
\begin{array}{r}
a_{1}-\alpha_{1}\left(a_{1}\right)=a_{1}-a_{1}=0 \in C_{1}^{D+}(X), \\
\left(a_{1}, a_{2}\right)-\alpha_{2}\left(a_{1}, a_{2}\right)=\left(a_{1}, a_{2}\right)-\left(a_{1}, a_{2}\right)+\left(a_{1}, a_{1}\right)=\left(a_{1}, a_{1}\right) \in C_{2}^{D+}(X) .
\end{array}
$$


Suppose $c-\alpha_{n}(c) \in C_{n}^{D+}(X)$ for some $n$, and consider

$$
\begin{aligned}
& \left(a_{1}, \ldots, a_{n+1}\right)-\alpha_{n+1}\left(a_{1}, \ldots, a_{n+1}\right) \\
& =\left(a_{1}, \ldots, a_{n+1}\right)-\left(\alpha_{n}\left(a_{1}, \ldots, a_{n}\right), a_{n+1}\right)+\left(\alpha_{n}\left(a_{1}, \ldots, a_{n}\right), a_{n}\right) \\
& =\left(a_{1}, \ldots, a_{n+1}\right)-\left(\alpha_{n}\left(a_{1}, \ldots, a_{n}\right), a_{n+1}\right)-\left(a_{1}, \ldots, a_{n}, a_{n}\right)+\left(\alpha_{n}\left(a_{1}, \ldots, a_{n}\right), a_{n}\right) \\
& \quad+\left(a_{1}, \ldots, a_{n}, a_{n}\right) \\
& =\left(\left(a_{1}, \ldots, a_{n}\right)-\alpha_{n}\left(a_{1}, \ldots, a_{n}\right), a_{n+1}\right)-\left(\left(a_{1}, \ldots, a_{n}\right)-\alpha_{n}\left(a_{1}, \ldots, a_{n}\right), a_{n}\right) \\
& +\left(a_{1}, \ldots, a_{n}, a_{n}\right) \in C_{n}^{D+}(X) .
\end{aligned}
$$

In order to show that $w_{n} \circ u_{n}=\mathrm{id}$, choose $c=\left(a_{1}, \ldots, a_{i}, a_{i+1}, \ldots, a_{n}\right) \in C_{n}^{D+}(X)$, where $a_{i}=a_{i+1}$. It suffices to prove that $\alpha_{n}(c)=0$. In fact,

$$
\alpha_{n}(c)=\left(a_{1}, a_{2}-a_{1}, \ldots, a_{i+1}-a_{i}, \ldots, a_{n}-a_{n-1}\right)=0 .
$$

Next we show that $w_{n}: C_{n}^{R+}(X) \rightarrow C_{n}^{D+}(X)$ is a chain map. We need the two equalities below $(n \geq 2)$ :

$$
\begin{aligned}
& \alpha_{n}\left(d_{1}\left(a_{1}, \ldots, a_{n}\right), a_{n}\right)=\alpha_{n}\left(-\left(a_{2}, \ldots, a_{n}, a_{n}\right)+\cdots+(-1)^{n}\left(a_{1}, \ldots, a_{n}\right)\right) \\
&=(-1)^{n} \alpha_{n}\left(a_{1}, \ldots, a_{n}\right), \\
& \alpha_{n}\left(d_{2}\left(a_{1}, \ldots, a_{n}\right), a_{n}\right)=\alpha_{n}\left(\sum_{i=1}^{n}(-1)^{i}\left(a_{1} * a_{i}, \ldots, a_{i-1} * a_{i}, a_{i+1}, \ldots, a_{n}, a_{n}\right)\right) \\
&=(-1)^{n} \alpha_{n}\left(\left(a_{1}, \ldots, a_{n}\right) * a_{n}\right) .
\end{aligned}
$$

Now we show that $\partial_{n+1}^{+} \alpha_{n+1}-\alpha_{n} \partial_{n+1}^{+}=0$. First note that

$$
\partial_{2}^{+} \alpha_{2}\left(a_{1}, a_{2}\right)=-\left(a_{2}\right)-\left(a_{2}\right)+\left(a_{1}\right)+\left(a_{1} * a_{2}\right)=\alpha_{1} \partial_{2}^{+}\left(a_{1}, a_{2}\right) .
$$

Assuming $\partial_{n+1}^{+} \alpha_{n+1}-\alpha_{n} \partial_{n+1}^{+}=0$ holds for some $n \geq 2$, one computes

$$
\begin{aligned}
\partial_{n+1}^{+} \alpha_{n+1} & \left(a_{1}, \ldots, a_{n+1}\right)-\alpha_{n} \partial_{n+1}^{+}\left(a_{1}, \ldots, a_{n+1}\right) \\
=\partial_{n+1}^{+} & \left(\left(\alpha_{n}\left(a_{1}, \ldots, a_{n}\right), a_{n+1}\right)-\left(\alpha_{n}\left(a_{1}, \ldots, a_{n}\right), a_{n}\right)\right) \\
& -\alpha_{n}\left(\left(\partial_{n}^{+}\left(a_{1}, \ldots, a_{n}\right), a_{n+1}\right)+(-1)^{n+1}\left(a_{1}, \ldots, a_{n}\right)\right. \\
& \left.+(-1)^{n+1}\left(a_{1}, \ldots, a_{n}\right) * a_{n+1}\right) \\
=\left(\partial_{n}^{+}\right. & \left.\alpha_{n}\left(a_{1}, \ldots, a_{n}\right), a_{n+1}\right)+(-1)^{n+1} \alpha_{n}\left(a_{1}, \ldots, a_{n}\right) \\
& +(-1)^{n+1} \alpha_{n}\left(a_{1}, \ldots, a_{n}\right) * a_{n+1}-\left(\partial_{n}^{+} \alpha_{n}\left(a_{1}, \ldots, a_{n}\right), a_{n}\right) \\
& -(-1)^{n+1} \alpha_{n}\left(a_{1}, \ldots, a_{n}\right)-(-1)^{n+1} \alpha_{n}\left(a_{1}, \ldots, a_{n}\right) * a_{n} \\
& -\left(\alpha_{n-1} \partial_{n}^{+}\left(a_{1}, \ldots, a_{n}\right), a_{n+1}\right)-\left(\alpha_{n-1} \partial_{n}^{+}\left(a_{1}, \ldots, a_{n}\right), l\left(\partial_{n}^{+}\left(a_{1}, \ldots, a_{n}\right)\right)\right) \\
& -(-1)^{n+1} \alpha_{n}\left(a_{1}, \ldots, a_{n}\right)-(-1)^{n+1} \alpha_{n}\left(\left(a_{1}, \ldots, a_{n}\right) * a_{n+1}\right)
\end{aligned}
$$




$$
\begin{aligned}
&=-\left(\alpha_{n-1} \partial_{n}^{+}\left(a_{1}, \ldots, a_{n}\right), a_{n}\right)-(-1)^{n+1} \alpha_{n}\left(a_{1}, \ldots, a_{n}\right) \\
& \quad-(-1)^{n+1} \alpha_{n}\left(a_{1}, \ldots, a_{n}\right) * a_{n}-\left(\alpha_{n-1} \partial_{n}^{+}\left(a_{1}, \ldots, a_{n}\right), l\left(\partial_{n}^{+}\left(a_{1}, \ldots, a_{n}\right)\right)\right) \\
&=-\alpha_{n}\left(\partial_{n}^{+}\left(a_{1}, \ldots, a_{n}\right), a_{n}\right)-(-1)^{n+1} \alpha_{n}\left(a_{1}, \ldots, a_{n}\right) \quad-(-1)^{n+1} \alpha_{n}\left(a_{1}, \ldots, a_{n}\right) * a_{n} \\
& \quad-(-1)^{n+1} \alpha_{n}\left(a_{1}, \ldots, a_{n}\right) * a_{n} \\
&=-\alpha_{n}\left(\left(d_{1}+d_{2}\right)\left(a_{1}, \ldots, a_{n}\right), a_{n}\right)-(-1)^{n+1} \alpha_{n}\left(a_{1}, \ldots, a_{n}\right) \quad-(-1)^{n+1} \alpha_{n}\left(a_{1}, \ldots, a_{n}\right)-(-1)^{n+1} \alpha_{n}\left(a_{1}, \ldots, a_{n}\right) * a_{n} \\
&=-(-1)^{n} \alpha_{n}\left(a_{1}, \ldots, a_{n}\right)-\left(-1, a_{n}\right) * a_{n} \quad-(-1, \ldots \\
&=0
\end{aligned}
$$

This completes the proof.

Now we investigate $H_{1}^{Q+}(X)$ and $H_{2}^{Q+}(X)$ for a general quandle $X$. Similar results for quandle homology groups can be found in [5; 21]. Assume $X=\left\{a_{1}, \ldots, a_{n}\right\}$. According to the definitions of $d_{1}$ and $d_{2}$ we have $Z_{1}^{Q+}(X)=C_{1}^{Q+}(X)=C_{1}^{R+}(X)$, ie the free abelian group generated by $\left\{a_{1}, \ldots, a_{n}\right\}$. Since $\partial_{2}^{+}(a, b)=-b-b+a+a * b$, we conclude that

$$
H_{1}^{Q+}(X) \cong\left\{a_{1}, \ldots, a_{n} \mid a_{i} * a_{j}=2 a_{j}-a_{i}\right\}
$$

Proposition 4.4 We have $H_{1}^{Q+}\left(T_{n}\right) \cong \mathbb{Z} \oplus\left(\bigoplus_{n-1} \mathbb{Z}_{2}\right)$ and $H_{1}^{Q+}\left(R_{n}\right) \cong \mathbb{Z} \oplus \mathbb{Z}_{n}$.

Proof According to the analysis above, we have

$$
\begin{aligned}
H_{1}^{Q+}\left(T_{n}\right) \cong\left\{a_{1}, \ldots, a_{n} \mid 2 a_{i}=2 a_{j}\right\} & \cong\left\{a_{1}, a_{2}-a_{1}, \ldots, a_{n}-a_{1} \mid 2\left(a_{i}-a_{1}\right)=0\right\} \\
& \cong \mathbb{Z} \oplus\left(\bigoplus_{n-1} \mathbb{Z}_{2}\right) .
\end{aligned}
$$

For the dihedral quandle $R_{n}=\left\{a_{0}, \ldots, a_{n-1}\right\}$ with quandle operations $a_{i} * a_{j}=$ $a_{2 j-i(\bmod n)}$, we have

$$
\begin{aligned}
& H_{1}^{Q+}\left(R_{n}\right) \cong\left\{a_{0}, \ldots, a_{n-1} \mid a_{2 j-i(\bmod n)}=2 a_{j}-a_{i}\right\} \\
& \cong\left\{a_{0}, a_{1}-a_{0} \mid n\left(a_{1}-a_{0}\right)=0\right\} \cong \mathbb{Z} \oplus \mathbb{Z}_{n} .
\end{aligned}
$$

This completes the proof.

Next we study the second positive degeneration homology $H_{2}^{D+}(X)$. Given a quandle $X$ and $\{a, b\} \in X$, we define $a \sim b$ if there exists some elements $a_{1}, \ldots, a_{n}$ of $X$ such that $b=\left(\cdots\left(\left(a *^{\varepsilon_{1}} a_{1}\right) *^{\varepsilon_{2}} a_{2}\right) \cdots\right) *^{\varepsilon_{n}} a_{n}$, where $\varepsilon_{i} \in\{ \pm 1\}$. The orbits 
of $X$ are defined to be the set of equivalence classes of $X$ by $\sim$. We denote it by $\operatorname{Orb}(X)$, and as usual the number of elements in $\operatorname{Orb}(X)$ is denoted by $|\operatorname{Orb}(X)|$. Since $\partial^{+}(a, a)=-a-a+a+a=0$,

$$
\begin{aligned}
\partial^{+}(a, a, b) & =-2(a, b)+(a, b)+(a, b)-(a, a)-(a * b, a * b) \\
& =-(a, a)-(a * b, a * b),
\end{aligned}
$$

and

$$
\partial^{+}(a, b, b)=-2(b, b)+(a, b)+(a * b, b)-(a, b)-(a * b, b)=-2(b, b),
$$

so combining this with Theorem 4.3, it follows that:

Proposition 4.5 We have isomorphisms

$$
H_{2}^{D+}(X) \cong \bigoplus_{|\operatorname{Orb}(X)|} \mathbb{Z}_{2} \text { and } H_{2}^{R+}(X) \cong H_{2}^{Q+}(X) \bigoplus\left(\bigoplus_{|\operatorname{Orb}(X)|} \mathbb{Z}_{2}\right)
$$

In the end of this section let us turn to the trivial quandle $T_{n}$. In quandle homology theory, the boundary operators of $T_{n}$ are trivial, therefore $H_{n}^{Q}\left(T_{n}\right) \cong C_{n}^{Q}\left(T_{n}\right)$. However in positive quandle homology theory, the boundary operators are not trivial in general. In fact we have the following proposition.

Proposition 4.6 We have

$$
H_{i}^{Q+}\left(T_{n}\right) \cong\left\{\begin{array}{ll}
\mathbb{Z} \oplus \bigoplus_{\mathbb{Z}_{2}} \quad i=1, \\
\bigoplus_{(n-1)^{i}}^{n-1} \mathbb{Z}_{2}
\end{array} \quad i \geq 2, \quad H_{Q+}^{i}\left(T_{n}\right) \cong\left\{\begin{array}{cc}
\mathbb{Z} & i=1, \\
\bigoplus_{(n-1)^{i-1}} \mathbb{Z}_{2} & i \geq 2 .
\end{array}\right.\right.
$$

Proof It suffices to compute $H_{i}^{Q+}\left(T_{n}\right)$ as then $H_{Q+}^{i}\left(T_{n}\right)$ can be deduced from the universal coefficient theorem. For the case $i=1$, the result follows from Proposition 4.4.

Now we show that $H_{2}^{Q+}\left(T_{n}\right) \cong \bigoplus_{(n-1)^{2}} \mathbb{Z}_{2}$. Recall that $T_{n}=\left\{a_{1}, \ldots, a_{n}\right\}$ with quandle operations $a_{i} * a_{j}=a_{i}$. Notice that

$$
\partial_{2}^{+}\left(a_{i}, a_{j}\right)=-2 a_{j}+a_{i}+a_{i} * a_{j}=2\left(a_{i}-a_{j}\right),
$$

therefore any element $\psi \in Z_{2}^{Q+}\left(T_{n}\right)$ can be written as $\psi=\sum_{i=1}^{n} c_{i} \psi_{i}$, where $\psi_{i}=\left(a_{i_{1}}, a_{i_{2}}\right)+\cdots+\left(a_{i_{k-1}}, a_{i_{k}}\right)+\left(a_{i_{k}}, a_{i_{1}}\right)$. It follows that $Z_{2}^{Q+}\left(T_{n}\right)$ is generated by

$$
\left\{\left(a_{i}, a_{j}\right)+\left(a_{j}, a_{i}\right),\left(a_{1}, a_{i}\right)+\left(a_{i}, a_{j}\right)+\left(a_{j}, a_{1}\right)\right\} \quad(1 \leq i<j \leq n)
$$


which is equivalent to

$$
\left\{\left(a_{1}, a_{i}\right)+\left(a_{i}, a_{j}\right)+\left(a_{j}, a_{1}\right)\right\} \quad(2 \leq i \leq j \leq n) .
$$

On the other hand, since

$$
\begin{aligned}
\partial^{+}\left(a_{i}, a_{j}, a_{k}\right) & =2\left(-\left(a_{j}, a_{k}\right)+\left(a_{i}, a_{k}\right)-\left(a_{i}, a_{j}\right)\right), \\
\partial^{+}\left(a_{i}, a_{j}, a_{i}\right) & =2\left(-\left(a_{j}, a_{i}\right)-\left(a_{i}, a_{j}\right)\right),
\end{aligned}
$$

we have

$$
\begin{aligned}
H_{2}^{Q+}\left(T_{n}\right) \cong & \left\{\left(a_{1}, a_{i}\right)+\left(a_{i}, a_{j}\right)+\left(a_{j}, a_{1}\right) \mid 2\left(\left(a_{i}, a_{j}\right)+\left(a_{j}, a_{i}\right)\right),\right. \\
& \left.2\left(\left(a_{i}, a_{j}\right)+\left(a_{j}, a_{k}\right)-\left(a_{i}, a_{k}\right)\right)\right\} \\
& \cong\left\{\left(a_{1}, a_{i}\right)+\left(a_{i}, a_{j}\right)+\left(a_{j}, a_{1}\right) \mid 2\left(\left(a_{1}, a_{i}\right)+\left(a_{i}, a_{j}\right)+\left(a_{j}, a_{1}\right)\right)\right\} \\
& \cong \bigoplus_{(n-1)^{2}} \mathbb{Z}_{2} .
\end{aligned}
$$

Similarly since $\partial_{i}^{+}=2 d_{1}$ for $C_{i}^{Q}\left(T_{n}\right)$, it is not difficult to observe that

$$
H_{i}^{Q+}\left(T_{n}\right) \cong\left\{\frac{1}{2}\left(\partial_{i+1}^{+}\left(a_{1}, a_{j_{1}}, \ldots, a_{j_{i}}\right)\right) \mid \partial_{i+1}^{+}\left(a_{1}, a_{j_{1}}, \ldots, a_{j_{i}}\right)\right\} \cong \underset{(n-1)^{i}}{\bigoplus} \mathbb{Z}_{2}
$$

here $2 \leq j_{k} \leq n$.

\section{Knot invariants derived from positive quandle cocycles}

\subsection{Positive quandle cocycle invariants for knots}

One of the most important applications of quandle cohomology groups is that one can define knot invariants via quandle 2-cocycles and knotted surface invariants via quandle 3-cocycles. In this section we will show that positive quandle 2-cocycles can also be used to define knot invariants, which is similar to the definition of quandle cocycle invariants introduced in [3].

Let $K$ be an oriented knot diagram and $X$ a finite quandle. Assume $G$ is an abelian group and $\phi \in Z_{Q+}^{2}(X ; G)$ is a positive quandle 2-cocycle. It is well known that all regions of $R^{2}-K$ can be colored with white and black in checkerboard fashion such that the unbounded region gets the white color. For each crossing point $\tau$ we can associate a sign $\epsilon(\tau)$ as in the figure below.

Let $\rho$ be a proper coloring of $K$ by $X$, ie a homomorphism from the fundamental quandle of $K$ to $X$. In other words, each arc of the diagram is labelled with an element of $X$. For each crossing point $\tau$, assume the over-arc and under-arcs at $\tau$ are colored 

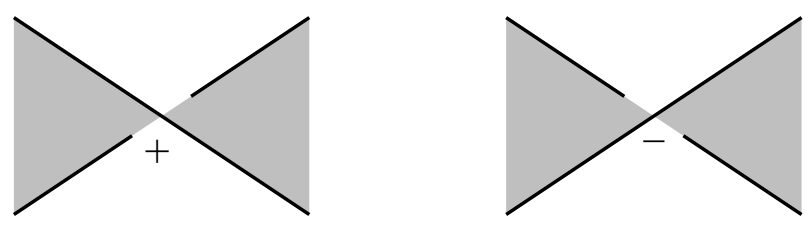

Figure 2: The signs of crossings

by $b$ and $a, a * b$ respectively; see Figure 1 . We consider a weight which is an element of $G$ as

$$
W_{\phi}(\tau, \rho)=\phi(a, b)^{\epsilon(\tau)},
$$

where $\epsilon(\tau)= \pm 1$ according to Figure 2. Then we define the positive quandle 2-cocycle invariant of $K$ to be

$$
\Phi_{\phi}(K)=\sum_{\rho} \prod_{\tau} W_{\phi}(\tau, \rho) \in \mathbb{Z} G,
$$

where $\rho$ runs all proper colorings of $K$ by $X$ and $\tau$ runs all crossing points of the diagram. Note that if the sign of the crossing $\epsilon(\tau)$ is replaced by the writhe of $\tau$, one obtains the state-sum (associated with a quandle 2-cocycle $\phi$ ) knot invariants defined by J S Carter et al in [3].

Theorem 5.1 The positive quandle 2-cocycle invariant $\Phi_{\phi}(K)$ is preserved under Reidemeister moves. If a pair of positive quandle 2-cocycles $\phi_{1}$ and $\phi_{2}$ are cohomologous, then $\Phi_{\phi_{1}}(K)=\Phi_{\phi_{2}}(K)$. In particular if $\phi$ is a coboundary, we have $\Phi_{\phi}(K)=\sum_{\mathrm{Col}_{X}(K)} 1$.

Proof First we prove that $\Phi_{\phi}(K)$ is invariant under Reidemeister moves. In [28], M Polyak proved that all the classical Reidemeister moves can be realized by a generating set of four Reidemeister moves: $\left\{\Omega_{1 a}, \Omega_{1 b}, \Omega_{2 a}, \Omega_{3 a}\right\}$; see Figure 3. Hence it suffices to show that $\Phi_{\phi}(K)$ is invariant under $\Omega_{1 a}, \Omega_{1 b}, \Omega_{2 a}$ and $\Omega_{3 a}$.
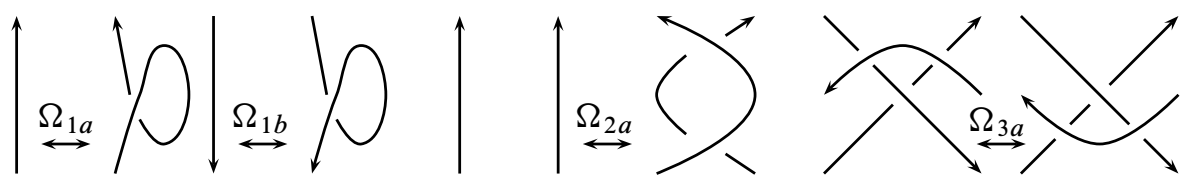

Figure 3: Reidemeister moves

$\boldsymbol{\Omega}_{1 \boldsymbol{a}}$ and $\boldsymbol{\Omega}_{\mathbf{1} \boldsymbol{b}}$ The weight assigned to the crossing point in $\Omega_{1 a}$ or $\Omega_{1 b}$ is of the form $\phi(a, a)^{ \pm 1}$. According to the definition of positive quandle cocycle we have $\phi(a, a)^{ \pm 1}=1$. 
$\boldsymbol{\Omega}_{\mathbf{2} a}$ Assume the two arcs on the left side are colored by $a, b$ respectively. Then the sum of the weights of the two crossing points on the right side is $\phi(b, a) \phi(b, a)^{-1}=1$.

$\boldsymbol{\Omega}_{\mathbf{3} \boldsymbol{a}}$ Without loss of generality, we assume the top region on both sides is colored white. Under this assumption the signs of each crossing are shown in the figure below.

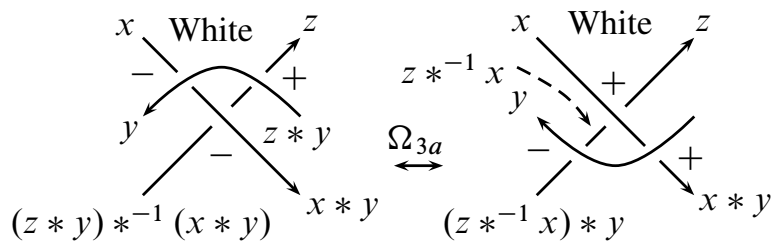

Figure 4: Proper colorings under $\Omega_{3 a}$

In order to show that $\Phi_{\phi}(K)$ is invariant under $\Omega_{3 a}$, it is sufficient to prove that $\phi(x, y)^{-1} \phi(z, y) \phi\left((z * y) *^{-1}(x * y), x * y\right)^{-1}=\phi\left(z *^{-1} x, y\right)^{-1} \phi\left(z *^{-1} x, x\right) \phi(x, y)$. Note that $(z * y) *^{-1}(x * y)=\left(z *^{-1} x\right) * y$. Put $(a, b, c)=\left(z *^{-1} x, x, y\right)$ and compare the equation with the positive quandle 2-cocycle condition (note that the equation is written in multiplicative notation here); the result follows.

In order to finish the proof it suffices to show that $\Phi_{\phi}(K)=\sum_{\operatorname{Col}_{X}(K)} 1$ if $\phi$ is a coboundary. Assume $\phi=\delta_{+}^{1} \varphi$ for some $\varphi \in C_{Q+}^{1}(X ; G)$. Then

$$
\begin{aligned}
\phi(a, b)=\delta_{+}^{1} \varphi(a, b)=\varphi\left(\partial_{2}^{+}(a, b)\right) & =\varphi(-2(b)+(a)+(a * b)) \\
& =\varphi(b)^{-2} \varphi(a) \varphi(a * b) \in G .
\end{aligned}
$$

First let us consider the simplest case: we assume the knot diagram is alternating. Therefore all crossings have the same sign. Without loss of generality all the crossings are assumed to be positive. In this case for a given arc $\lambda$ of the knot diagram, there exists only one crossing such that $\lambda$ is the over-arc at this crossing. On the other hand, this arc is the under-arc at two crossings. For a fixed proper coloring $\rho$, suppose the labelled element of $\lambda$ is $a \in X$. Then the contribution of $\lambda$ to $\prod_{\tau} W_{\phi}(\tau, \rho)$ comes from the three crossing points that $\lambda$ involved, which equals $\varphi(a)^{-2} \varphi(a) \varphi(a)=1$. It follows that $\prod_{\tau} W_{\phi}(\tau, \rho)=1$, hence $\Phi_{\phi}(K)=\sum_{\mathrm{Col}_{X}(K)} 1$. The proof of the nonalternating case is analogous to the alternating case. In fact it suffices to notice that if an arc $\lambda$ is the over-arc at several crossings, then the signs of these crossings are alternating. It is not difficult to find that the contribution of $\lambda$ to $\prod_{\tau} W_{\phi}(\tau, \rho)$ is still trivial. The proof is finished.

Recall that in quandle cohomology theory $H_{Q}^{2}\left(R_{3}\right)=0$, it means quandle 2-cocycle invariant of $R_{3}$ cannot offer any more information than the Fox 3-colorings. In fact it 
was pointed out in [3] that all knots have trivial quandle 2-cocycle invariants with any dihedral quandle $R_{n}$ and any quandle 2-cocycle. We remark that although quandle 2-cocycle invariants of $R_{n}$ are trivial, some quandle 3-cocycle of $H_{Q}^{3}\left(R_{3} ; \mathbb{Z}_{3}\right)$ can be used to distinguish the trefoil and its mirror image; see Rourke and Sanderson [34].

Proposition 5.2 All knots have trivial positive quandle 2-cocycle invariants with any dihedral quandle $R_{n}$, associated with any positive quandle 2 -cocycle $\phi \in Z_{Q+}^{2}\left(R_{n}\right)$.

Proof If $n$ is even, according to the coloring rule at each crossing point, for each colored knot diagram all the assigned elements have the same parity. If all assigned elements are even, then by replacing the assigned element $i$ with $i / 2$ we obtain a proper coloring of $R_{n / 2}$. Consider the element $\phi^{\prime}$ of $Z_{Q+}^{2}\left(R_{n / 2}\right)$ defined by $\phi^{\prime}(i, j)=$ $\phi(2 i, 2 j)$. Then $\Phi_{\phi^{\prime}}(K)$ with $R_{n / 2}$ is nontrivial if $\Phi_{\phi}(K)$ with $R_{n}$ is nontrivial. If all assigned elements are odd, then one obtains a proper coloring with $R_{n / 2}$ by replacing each labelled element $i$ with $(i-1) / 2$. Similarly if $\Phi_{\phi}(K)$ with $R_{n}$ is nontrivial then $\Phi_{\phi^{\prime \prime}}(K)$ with $R_{n / 2}$ is also nontrivial, where $\phi^{\prime \prime}(i, j)=\phi(2 i+1,2 j+1)$. Therefore it is sufficient to consider the case of odd $n$.

If $n$ is odd, it suffices to prove that the free part of $H_{Q+}^{2}\left(R_{n}\right)$ is 0 . This follows from a general fact: $\Phi_{\phi}(K)$ is trivial if $\phi$ has finite order in $H_{Q+}^{2}(X)$. In fact assume $k \phi=0 \in H_{Q+}^{2}(X)$; then $\prod_{\tau} W_{k \phi}(\tau, \rho)=0$. In other words,

$$
\prod_{\tau} k \phi(a, b)^{\epsilon(\tau)}=k\left(\prod_{\tau} \phi(a, b)^{\epsilon(\tau)}\right)=0 .
$$

Since we are working with coefficients in $\mathbb{Z}$, it follows that $\prod_{\tau} \phi(a, b)^{\epsilon(\tau)}=0$.

Assume the free part of $H_{Q+}^{2}\left(R_{n}\right)$ is not equal to 0 . It follows that the free part of $H_{2}^{Q+}\left(R_{n}\right) \neq 0$. Replacing the coefficient $\mathbb{Z}$ by $\mathbb{Z}_{2}$ one concludes that $H_{2}^{Q+}\left(R_{n} ; \mathbb{Z}_{2}\right)$ contains $\mathbb{Z}_{2}$ as a summand. By Proposition 3.3 we have $H_{2}^{Q}\left(R_{n} ; \mathbb{Z}_{2}\right)=\mathbb{Z}_{2} \oplus$ other. However since $H_{2}^{Q}\left(R_{n} ; \mathbb{Z}\right)=0$ by [5] and $H_{1}^{Q}\left(R_{n} ; \mathbb{Z}\right)=\mathbb{Z}$, the universal coefficient theorem tells us that $H_{2}^{Q}\left(R_{n} ; \mathbb{Z}_{2}\right)=0$. The proof is finished.

Now we give a nontrivial example of positive quandle 2-cocycle invariant. With the matrix of a finite quandle introduced in [17], the quandle $S_{4}$ contains four elements $\{0,1,2,3\}$ with quandle operations

$$
\left[\begin{array}{llll}
0 & 2 & 3 & 1 \\
3 & 1 & 0 & 2 \\
1 & 3 & 2 & 0 \\
2 & 0 & 1 & 3
\end{array}\right],
$$


where the entry in row $i$ column $j$ denotes $(i-1) *(j-1)(1 \leq i, j \leq 4)$. Choose a positive quandle 2 -cocycle

$$
\phi=\chi_{(0,1)}+\chi_{(1,0)}+\chi_{(2,0)}+\chi_{(0,2)}+\chi_{(1,2)}+\chi_{(2,1)} \in H_{Q+}^{2}\left(S_{4} ; \mathbb{Z}_{2}\right) ;
$$

it was proved in [3] that $\Phi_{\phi}\left(3_{1}\right)=\Phi_{\phi}\left(4_{1}\right)=\sum_{4} 0+\sum_{12} 1$.

We end this subsection with some remarks on the positive quandle 2-cocycle invariants with trivial quandles. First note that for $T_{n}$ and for any knot diagram there exist exactly $n$ trivial proper colorings. By the definition of \pm quandle homology groups we cannot obtain any new information from the \pm quandle cocycle invariants. However it was pointed out in [3] that for any $\phi \in H_{Q}^{2}\left(T_{n}\right)$ and any link $L$, the quandle 2cocycle invariant $\Phi_{\phi}(L)$ is a function of pairwise linking numbers. For example $\phi=\chi_{\left(a_{1}, a_{2}\right)} \in H_{Q}^{2}\left(T_{2}\right)$ can be used to distinguish the Hopf link from the trivial link. Since we have $H_{Q+}^{2}\left(T_{2}\right) \cong \mathbb{Z}_{2}$ with generator $\phi=\chi_{\left(a_{1}, a_{2}\right)}-\chi_{\left(a_{2}, a_{1}\right)}$, one obtains that $\Phi_{\phi}(L)$ is trivial for any link $L$. In order to obtain some information from the link, we can work with $\mathbb{Z}_{2}$ coefficients. In this way we can obtain parity information of the pairwise linking numbers. For example, a link $L=K_{1} \cup \cdots \cup K_{m}$ is a proper link, ie $\sum_{j \neq i} \operatorname{lk}\left(K_{i}, K_{j}\right)=0(\bmod 2)$ for any $1 \leq i \leq m$ if and only if $\sum_{\rho_{1, m-1}} \prod_{\tau} W_{\phi=\chi_{\left(a_{1}, a_{2}\right)}}\left(\tau, \rho_{1, m-1}\right)=\sum_{m} 0$. Here $\mathbb{Z}_{2}=\{0,1\}$ and $\rho_{1, m-1}$ denotes the set of proper colorings which assign one component with $a_{1}$ and the rest with $a_{2}$. This result mainly follows from the fact that $H_{Q+}^{2}\left(X ; \mathbb{Z}_{2}\right) \cong H_{Q}^{2}\left(X ; \mathbb{Z}_{2}\right)$. From this viewpoint, for $T_{n}$ it seems that the positive quandle 2-cocycle invariant is a sort of $\mathbb{Z}_{2}$-version of the quandle 2 -cocycle invariant. Later, in the final section, we will show that this is not the case.

\subsection{Positive quandle cocycle invariants for knotted surfaces}

In this subsection, given a positive quandle 3-cocycle we will define a state-sum invariant for knotted surfaces in $R^{4}$. First we will give a short review of the background of knotted surfaces in $R^{4}$. The readers are referred to Carter and Saito [7] and Carter, Kamada and Saito [8] for more details.

By a knotted surface we mean an embedding $f$ of a closed oriented surface $F$ into $R^{4}$. Sometimes we also call the image $f(F)$ a knotted surface and denote it by $F$ for convenience. In particular when $F=S^{2}$ we name it a $2-k n o t$. Two knotted surfaces are equivalent if there exists an orientation preserving automorphism of $R^{4}$ which takes one knotted surface to the other. Similar to the knot diagram in knot theory, we usually study knotted surfaces via the knotted surface diagrams. Let $p: R^{4} \rightarrow R^{3}$ be the orthogonal projection from $R^{4}$ onto $R^{3}$. We may deform $f(F)$ slightly such that $p \circ f(F)$ is in a general position. Then $p \circ f(F)$ is called a knotted surface diagram. 
We must notice that a knotted surface diagram does not just mean an immersed surface in $R^{3}$. First there exist double points, triple points and branch points in $p \circ f(F)$. However it is well known that $f(F)$ can be isotoped into a new position such that the projection contains no branch points; see Carter and Saito [6] and Giller [15]. Second, a knot diagram can be regarded as a 4-valent planar graph with some overunder information on each vertex. Hence a knotted surface diagram also contains the information of the over-sheet and under-sheet along the double curves. In other words, a knotted surface diagram is obtained from the projection by removing small open neighborhoods of the under-sheets along double curves.

Similar to the definition of the knot invariant $\operatorname{Col}_{X}(K)$, we can define an integer-valued knotted surface invariant with a given quandle $X$. The main idea is to use the elements of $X$ to color the regions of the broken surface diagram according to some rules at double curves. See the figure below, where $\vec{n}$ denotes the normal vector of the knotted surface diagram.

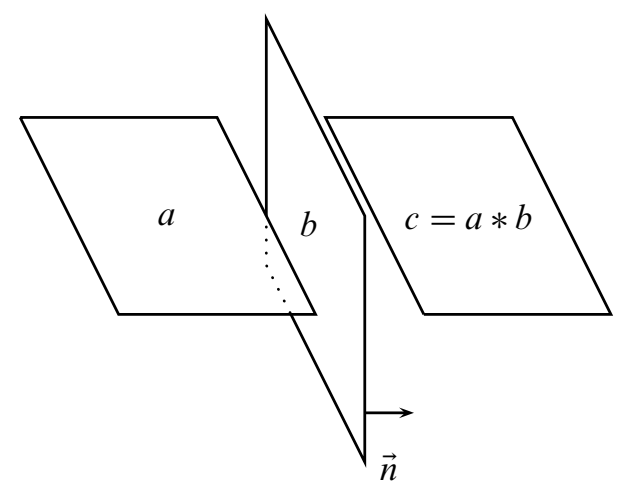

Figure 5: Coloring rules at a double curve

It is not difficult to check that the rule above is well defined at each triple point [3]. Recall that different knotted surface diagrams represent the same knotted surface if and only if one of them can be achieved from the other by a finite sequence of Roseman moves; see Roseman [33]. Similar to the proper coloring of knot diagrams, the number of the coloring satisfying the condition above is invariant under the Roseman moves, hence is a knotted surface invariant. We use $\operatorname{Col}_{X}(F)$ to denote it.

The main idea of defining a knotted surface invariant with a positive quandle 3-cocycle is analogous to the definition of the quandle 3-cocycle invariant proposed in [3]. As a generalization of the counting invariant $\mathrm{Col}_{X}(F)$, we need to assign an invariant to each colored knotted surface diagram and then take the sum of them. The position of 
the triple point in a knotted surface diagram is analogous to that of the crossing point in a knot diagram. Therefore this invariant can be obtained by assigning a weight to each triple point of the colored diagram.

Let $F$ be a knotted surface diagram and $X$ a finite quandle. Assume $G$ is an abelian group and $\theta \in Z_{Q+}^{3}(X ; G)$ is a positive quandle 3 -cocycle. Consider the shadow of the diagram $F$, which is the immersed surface in $R^{3}$ without removing a neighborhood along double curves. The shadow separates $R^{3}$ into several regions. It is not difficult to observe that we can use white and black to color these regions in 3-dimensional checkerboard fashion, ie adjacent regions are colored with different colors. As before we assume that the unique unbounded region is colored white. For each triple point $\tau$ we can associate a sign $\epsilon(\tau)$ according to the figure below ( $\mathrm{W}=$ white, $\mathrm{B}=$ black).

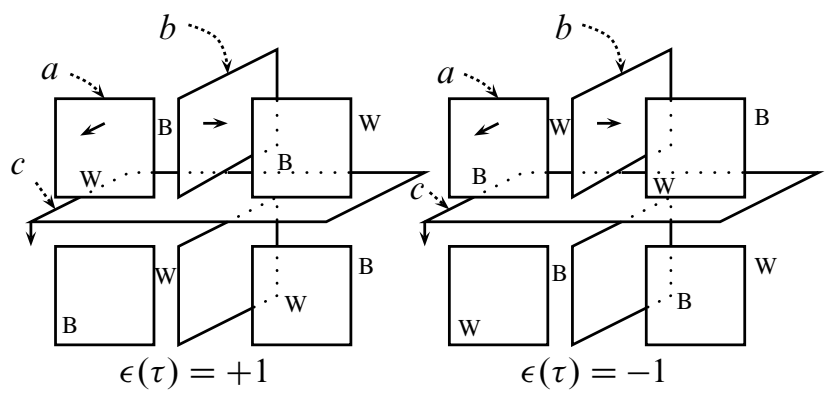

Figure 6: Signs of triple points

Let $\rho$ denote a coloring of $F$ by $X$. Assume $\tau$ is a triple point of $F$ and the bottom, middle, top sheets around the octant from which all normal vectors point outwards are colored by $a, b, c$ respectively; see the figure above. Note that the sign of the triple point used here does not depend on the orientation of the surface. We associate a weight at the triple point $\tau$ as

$$
W_{\theta}(\tau, \rho)=\theta(a, b, c)^{\epsilon(\tau)} \in G .
$$

Now we can define the positive quandle 3-cocycle invariant of the knotted surface $F$ associated with $\theta$ to be

$$
\Theta_{\theta}(F)=\sum_{\rho} \prod_{\tau} W_{\theta}(\tau, \rho) \in \mathbb{Z} G,
$$

where $\rho$ runs all colorings of $F$ by $X$ and $\tau$ runs all triple points of the diagram.

We remark that the sign of a triple point has another definition. Consider the normal vectors of the top, middle and bottom sheets. If the orientation in this order matches the orientation of $R^{3}$, we say this triple point is positive. Otherwise it is negative. 
Replace $\epsilon(\tau)$ with the sign of triple point defined in this way one obtains the state-sum invariants introduced in [3].

Theorem 5.3 The positive quandle 3-cocycle invariant $\Theta_{\theta}(F)$ is preserved under Roseman moves. If a pair of positive quandle 3-cocycles $\theta_{1}$ and $\theta_{2}$ are cohomologous, then $\Theta_{\theta_{1}}(F)=\Theta_{\theta_{2}}(F)$. In particular if $\theta$ is a coboundary, we have $\Theta_{\theta}(F)=$ $\sum_{\mathrm{Col}_{X}(F)} 1$.

Proof We summarize the proof. There are only three types of Roseman moves that involve triple points; see [3]. The first one creates or cancels a pair of triple points with oppositive signs, and the second one moves a branch point through a sheet. The contribution of the two triple points in the first case will cancel out, and the contribution of the triple point in the second case is trivial according to the definition of positive quandle cohomology groups. Thus it suffices to prove that $\Theta_{\theta}(F)$ is invariant under tetrahedral move. See the figures below.

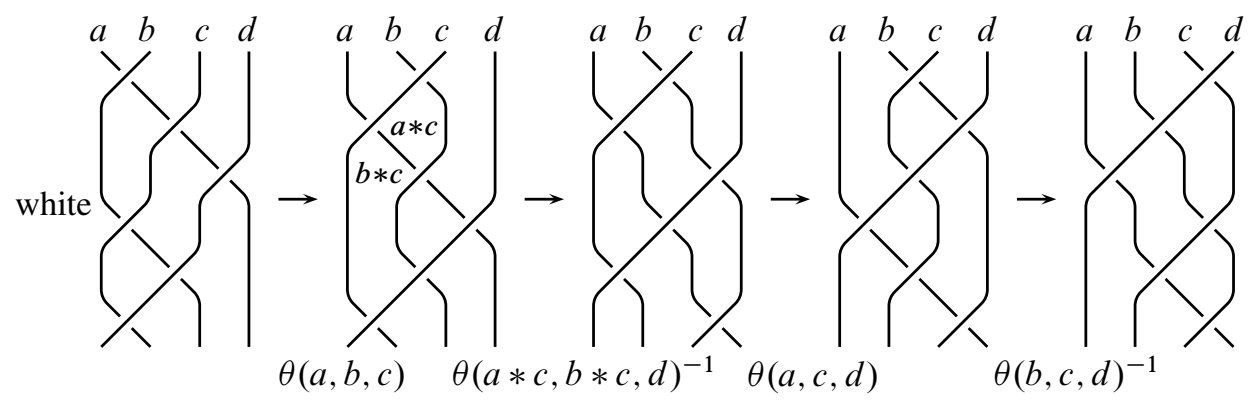

Figure 7: Left-hand side of the tetrahedral move

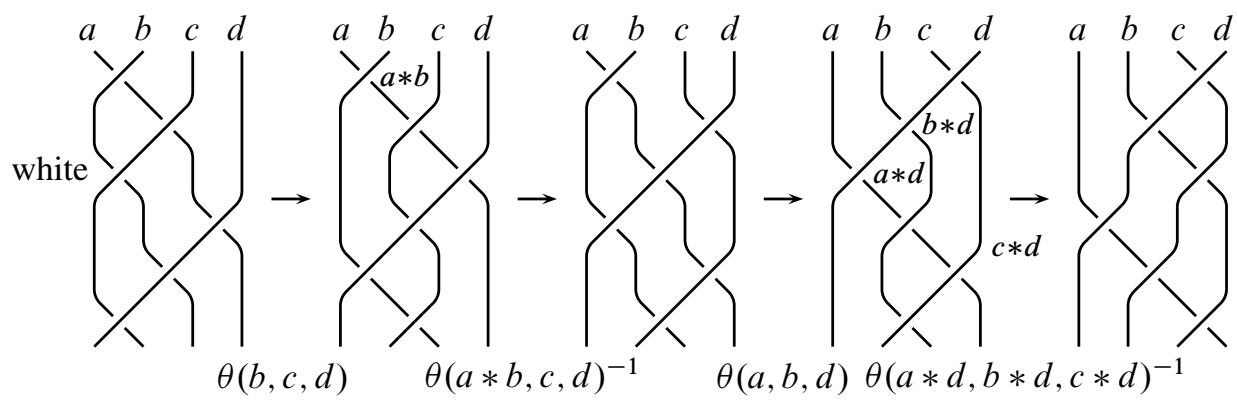

Figure 8: Right-hand side of the tetrahedral move

Here we use the movie description of knotted surface; see [7] for more detail. For example Figure 7 contains five slices of a knotted surface according to a fixed height function. Each slice consists of four sheets which are cross sections of four planes, 
and a pair of adjacent slices depict a triple point. Figures 7 and 8 correspond to the left-hand side and the right-hand side of the tetrahedral move. Without loss of generality, suppose the leftmost region of each slice has the white color, and other regions can be colored in checkerboard fashion. The left-hand side of the tetrahedral move contributes $\theta(a, b, c) \theta(a * c, b * c, d)^{-1} \theta(a, c, d) \theta(b, c, d)^{-1}$ to $\Theta_{\theta}(F)$, and the right side has the contribution $\theta(b, c, d) \theta(a * b, c, d)^{-1} \theta(a, b, d) \theta(a * d, b * d, c * d)^{-1}$. In order to prove that $\Theta_{\theta}(F)$ is invariant under the tetrahedral move, it suffices to show that

$$
\begin{aligned}
\theta(a, b, c) \theta(a * c, b * c, d)^{-1} \theta(a, c, d) \theta(b, c, d)^{-1} \theta(b, c, d)^{-1} \theta(a * b, c, d) \\
\cdot \theta(a, b, d)^{-1} \theta(a * d, b * d, c * d)=1 .
\end{aligned}
$$

Comparing the equation above with the positive quandle 3-cocycle condition (note that the equation is written in multiplicative notation at present), we find that the condition $\theta \in Z_{Q+}^{3}(X ; G)$ guarantees the invariance of $\Theta_{\theta}(F)$. Here we only list one case of the tetrahedral move; for other possible tetrahedral moves the invariance of $\Theta_{\theta}(F)$ can be proved in the same way.

Next we show that $\Theta_{\theta}(F)=\sum_{\operatorname{Col}_{X}(F)} 1$ if $\theta$ is a coboundary. As we mentioned before, we can choose a knotted surface diagram such that the shadow of it contains no branch points. The double point set of it is a 6-valent graph and each vertex corresponds to a triple point. Fix a coloring $\rho$. According to the assumption that $\theta$ is a coboundary, ie $\theta=\delta_{+}^{2} \phi$ for some $\phi \in C_{Q+}^{2}(X ; G)$, we have

$$
\begin{aligned}
\theta(a, b, c)=\delta_{+}^{2} \phi(a, b, c) & =\phi\left(\partial_{3}^{+}(a, b, c)\right) \\
& =\phi(b, c)^{-2} \phi(a, c) \phi(a * b, c) \phi(a, b)^{-1} \phi(a * c, b * c)^{-1} \in G .
\end{aligned}
$$

Consider the triple point $\tau$ on the left side of Figure 6, which has a weight $W_{\theta}(\tau, \rho)=$ $\theta(a, b, c)=\phi(b, c)^{-2} \phi(a, c) \phi(a * b, c) \phi(a, b)^{-1} \phi(a * c, b * c)^{-1}$. There are six edges adjacent to the triple point $\tau$ : two of them come from the intersection of the top sheet and the middle sheet, two of them come from the intersection of the middle sheet and the bottom sheet and the rest come from the intersection of the bottom sheet and the top sheet. We use $t m_{1}(\tau), t m_{2}(\tau), m b_{1}(\tau), m b_{2}(\tau), b t_{1}(\tau), b t_{2}(\tau)$ to denote these edges, where $t m_{i}(\tau)(i=1,2)$ denotes the two edges belonging to the intersection of the top sheet and the middle sheet, $m b_{i}(\tau)(i=1,2)$ denotes the two edges belonging to the intersection of the middle sheet and the bottom sheet and $b t_{i}(\tau)(i=1,2)$ denotes the two edges belonging to the intersection of the bottom sheet and the top sheet. The order of the two edges belonging to the intersection of two sheets matches the orientation of the normal vector of the third sheet. Then the contribution of $\tau$ to $\Theta_{\theta}(F)$ can be separated into six parts: $\phi(b, c)^{-1}, \phi(b, c)^{-1}, \phi(a, b)^{-1}, \phi(a * c, b * c)^{-1}, \phi(a, c), \phi(a * b, c)$. We assign these six parts to $t m_{1}(\tau), t m_{2}(\tau), m b_{1}(\tau), m b_{2}(\tau), b t_{1}(\tau), b t_{2}(\tau)$ respectively. 
Therefore the contribution of $\tau$ can be regarded as the product of the contribution of the six edges adjacent to $\tau$. We remark that the contribution of each edge can be read directly from Figure 5; the double line in Figure 5 has contribution $\phi(a, b)^{ \pm 1}$. Here the sign of \pm 1 is decided by the position of the two sheets. The sign is positive if the two sheets are the top sheet and the bottom sheet; for other cases the sign is negative. If the sign of the triple point is negative then all the contributions will take the inverse.

In order to show that $\Theta_{\theta}(F)$ is trivial, it is sufficient to prove that each edge obtains opposite contributions from the two endpoints of it. We continue our discussion in two cases depending on whether the two endpoints have the same sign or not.
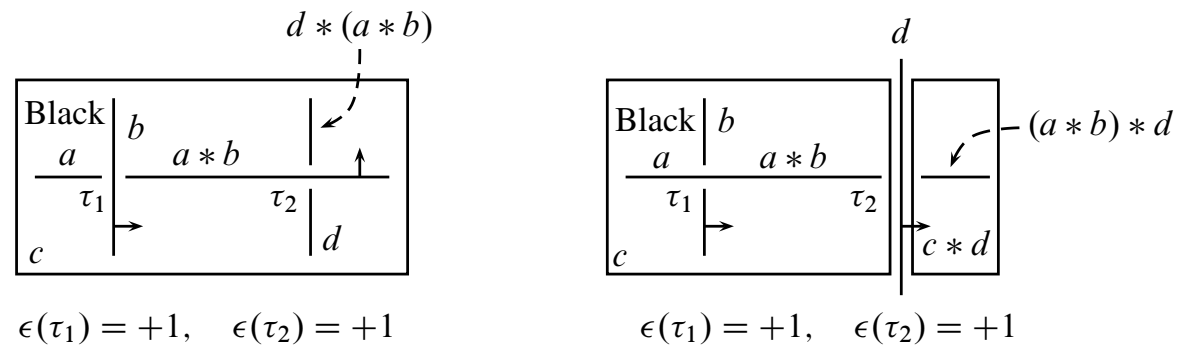

Figure 9: Two possibilities of adjacent triple points with the same sign

$\boldsymbol{\epsilon}\left(\tau_{1}\right)=+1$ and $\boldsymbol{\epsilon}\left(\tau_{2}\right)=+1$ There are two possibilities in this case. First consider the left side of Figure 9. There are two triple points $\tau_{1}$ and $\tau_{2}$ with the same sign. Without loss of generality we assume the sign is positive. The frame with color $c$ denotes the top sheet of $\tau_{1}$ and $\tau_{2}$, and the straight lines are cross sections between the middle sheet or bottom sheet with the top sheet. Since $W_{\theta}\left(\tau_{1}, \rho\right)=\theta(a, b, c)$ and $W_{\theta}\left(\tau_{2}, \rho\right)=\theta(d, a * b, c)$, the contribution from $\tau_{1}$ to the edge with color $a * b$ is $\phi(a * b, c)$ and that from $\tau_{2}$ is $\phi(a * b, c)^{-1}$. The negative sign comes from the fact that for the triple point $\tau_{2}$, the edge with color $a * b$ belongs to the intersection of the top sheet and the middle sheet. Hence the contributions from $\tau_{1}$ and $\tau_{2}$ to the edge between them cancel out. Considering the 6 -valent graph consisting of the double point set, it follows that the product of the contribution from each vertex to $\Theta_{\theta}(F)$ vanishes.

For the right side of Figure 9, we still have $\epsilon\left(\tau_{1}\right)=+1$ and $\epsilon\left(\tau_{2}\right)=+1$. Note that in this case the sheet with color $d$ is the top sheet of the triple point $\tau_{2}$. We have $W_{\theta}\left(\tau_{1}, \rho\right)=\theta(a, b, c)$ and $W_{\theta}\left(\tau_{2}, \rho\right)=\theta(a * b, c, d)$. Therefore the contribution from $\tau_{1}$ to the edge with color $a * b$ is $\phi(a * b, c)$ and the contribution from $\tau_{2}$ to the edge with color $a * b$ is $\phi(a * b, c)^{-1}$, since the edge with color $a * b$ belongs to the intersection of the middle sheet and the bottom sheet of $\tau_{2}$. Thus the contributions from $\tau_{1}$ and $\tau_{2}$ to the edge between them still cancel out. 


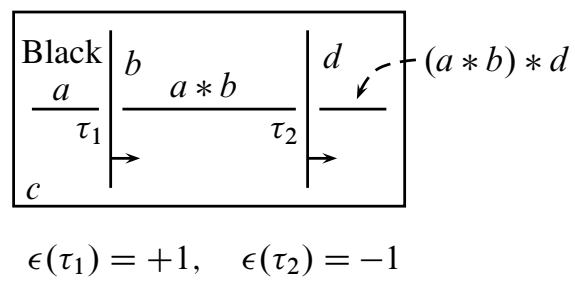

Figure 10: Adjacent triple points with different signs

$\boldsymbol{\epsilon}\left(\boldsymbol{\tau}_{1}\right)=+\mathbf{1}$ and $\boldsymbol{\epsilon}\left(\boldsymbol{\tau}_{2}\right)=-\mathbf{1}$ See Figure 10. We can read from the figure that $W_{\theta}\left(\tau_{1}, \rho\right)=\theta(a, b, c)$ and $W_{\theta}\left(\tau_{2}, \rho\right)=\theta(a * b, d, c)^{-1}$. As before the contribution from $\tau_{1}$ to the edge with color $a * b$ is $\phi(a * b, c)$. Meanwhile, due to $\epsilon\left(\tau_{2}\right)=-1$, the contribution from $\tau_{2}$ to the edge with color $a * b$ equals $\phi(a * b, c)^{-1}$. Hence in this case we still have $\prod_{\tau} W_{\theta}(\tau, \rho)=1$. The proof is finished.

Remark In quandle cohomology theory, a quandle 3-cocycle $\theta$ also can be used to define a state-sum invariant for knots via the shadow coloring. Given a knot diagram $K$ and a quandle $X$, a shadow coloring of $K$ by $X$ is a function from the set of arcs of $K$ and the regions separated by the shadow of $K$ to the quandle $X$, satisfying the coloring condition depicted below.
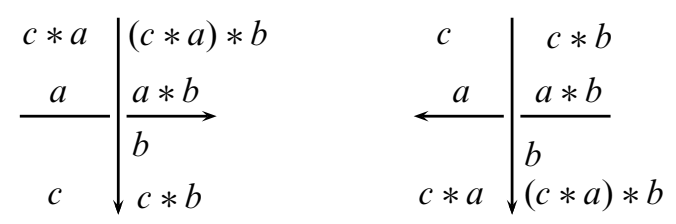

Figure 11: Shadow coloring at a crossing

It is not difficult to observe that shadow colorings are completely decided by the proper colorings on arcs and the color of one fixed region. Hence the number of shadow colorings does not offer any new information other than $\operatorname{Col}_{X}(K)$. Given a quandle 3-cocycle $\theta \in Z_{Q}^{3}(X ; G)$ one can associate a weight $W_{\theta}(\tau, \tilde{\rho})=\theta(c, a, b)^{w(\tau)}$ to the crossing point in Figure 11, where $w(\tau)$ means the writhe of the crossing and $\tilde{\rho}$ denotes a shadow coloring. Then the element $\Psi_{\theta}(K)=\sum_{\tilde{\rho}} \prod_{\tau} W_{\theta}(\tau, \tilde{\rho})$ of $\mathbb{Z} G$ defines a knot invariant, where $\tilde{\rho}$ runs all shadow colorings and $\tau$ runs all crossing points. It was pointed out in [34] that this state-sum invariant can be used to detect the chirality of the trefoil knot. An interesting question is how to define a knot invariant given a positive quandle 3 -cocycle.

Remark In [2] J S Carter et al generalized the quandle homology theory when the coefficient groups admit an Alexander quandle structure, for example when the coefficient 
group is $\mathbb{Z}\left[T, T^{-1}\right]$. A generalized version of cocycle invariants for knots and knotted surfaces, called the twisted quandle cocycle invaraints, were introduced with the use of the Alexander numbering of regions. Very recently the relation between the positive quandle homology and the twisted quandle homology was discussed by S Kamada, $\mathrm{V}$ Lebed and $\mathrm{K}$ Tanaka via shadow quandle cocycle invaraints [22]. By investigating the relation between the Alexander numbering and $\epsilon(\tau)$ S Kamada, V Lebed and K Tanaka proved that the cocycle invariants we introduced here are essentially equivalent to the invariants introduced in [2] when $T=-1$. On the other hand, we remark that S Kamada, V Lebed and K Tanaka's paper also offers an answer to the question above. The readers are referred to [22] for more details.

\section{On trivially colored crossing points}

We end this paper with two elementary examples which concern trivially colored crossing points. Given a knot diagram $K$ and a quandle $X$, choose a crossing point $\tau$ of the knot diagram. We say $\tau$ is a trivially colored crossing point if for any proper coloring of $K$ by $X$, the over-arc and the two under-arcs of $\tau$ are labelled with the same color. For example the crossing point involved in the first Reidemeister move is a trivially colored crossing point for any given quandle. As another instance, consider the crossing $\tau$ of the knot diagram below. If we take $X=R_{3}$, then the crossing $\tau$ is a trivially colored crossing point.

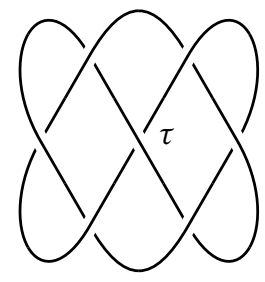

Figure 12: A trivially colored crossing point

There are two reasons for us to study trivially colored crossing points. The first comes from the Kauffman-Harary conjecture. L Kauffman and F Harary [16] conjectured that the minimum number of distinct colors that are needed to produce a nontrivial Fox $n$-coloring of a reduced alternating knot diagram $K$ with prime determinate $n$ equals the crossing number of $K$. In other words for any nontrivial Fox $n$-coloring of $K$, different arcs are assigned to different colors. In 2009 this conjecture was settled by T W Mattman and P Solis [24]. It means that for a given reduced alternating diagram with prime determinate $n$ and the quandle $R_{n}$, no crossing point of the knot diagram 
is trivially colored. However this conjecture does not hold if we ignore the condition of prime determinate. Consider for example the standard diagram of the connected sum of two reduced alternating knot diagrams which have prime determinate $m$ and $n$ respectively. Choose the quandle $R_{m n}$. Now there exists no Fox $m n$-coloring such that different arcs have different colors, but for each crossing point there exists a proper coloring such that this cross point is nontrivially colored. It is possible to extend the range of knots in the Kauffman-Harary conjecture by replacing the heterogeneity of the coloring with the nonexistence of trivially colored crossing points.

The second motivation of investigating trivially colored crossing points arises from the \pm quandle 2-cocycle invariants. Recall the definition of \pm quandle cohomology groups: in order for the 2-cocycle invariant to be preserved under the first Reidemeister move we put $\phi(a, a)=1$. In this way the first Reidemeister move has no effect on the 2-cocycle invariant, but the disadvantage is the information on trivially colored crossing points is also lost. For instance if a crossing point $\tau$ of a knot diagram $K$ is a trivially colored crossing point (associated with $X$ ), then $W_{\phi}(\tau, \rho)=1$ for any 2-cocycle $\phi$ and proper coloring $\rho$. Hence it has no contribution to the cocycle invariant.

The first example we want to discuss are the Borromean rings. The Borromean rings are a nontrivial 3-component link with trivial proper sublinks. That the Borromean rings form a nontrivial link follows from the fact that one component of the Borromean rings represents a commutator of the fundamental group of the complement of the other two components; see Rolfsen [32]. Let $X=T_{n}$. As we mentioned before, the quandle 2-cocycle of a link is a function of pairwise linking numbers [3]. Since the pairwise linking numbers of the Borromean rings are all trivial, it follows that the quandle 2-cocycle invariant cannot distinguish the Borromean rings from the trivial link. However we can use a refinement of the positive quandle 2-cocycle invariant to show that the Borromean rings arises nontrivial.

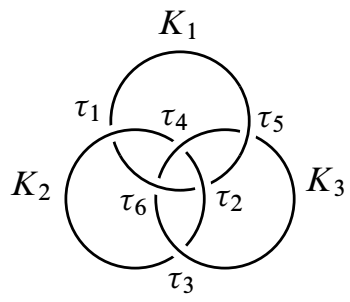

Figure 13: The Borromean rings

Let $K_{1}, K_{2}, K_{3}$ denote the three components of the Borromean rings and $\tau_{i}$ ( $1 \leq$ $i \leq 6)$ denote the crossing points of it. See the figure above. According to the 
definition of $\epsilon\left(\tau_{i}\right)$ we used in Section 5, we have $\epsilon\left(\tau_{i}\right)=+1 \quad(1 \leq i \leq 6)$. Take $\phi=\chi_{\left(a_{1}, a_{2}\right)}+\chi_{\left(a_{2}, a_{1}\right)} \in Z_{Q+}^{2}\left(T_{2} ; \mathbb{Z}_{4}\right)$, and consider the element

$$
\begin{aligned}
\tilde{\Phi}_{\phi}(\mathrm{BL})=\sum_{\rho}\left(t_{1}^{W_{\phi}\left(\tau_{1}, \rho\right)+W_{\phi}\left(\tau_{2}, \rho\right)} t_{2}^{W_{\phi}\left(\tau_{3}, \rho\right)+W_{\phi}\left(\tau_{4}, \rho\right)} t_{3}^{W_{\phi}\left(\tau_{5}, \rho\right)+W_{\phi}\left(\tau_{6}, \rho\right)}\right) & \\
& \in \mathbb{Z}\left[t_{1}, t_{2}, t_{3}\right] /\left(t_{1}^{4}=t_{2}^{4}=t_{3}^{4}=1\right),
\end{aligned}
$$

where $W_{\phi}\left(\tau_{i}, \rho\right)$ is the weight associated to the crossing $\tau_{i}$ and $\rho$ runs all proper colorings of the diagram in Figure 13 by $T_{2}$. In general for a diagram of a 3-component link $L=K_{1} \cup K_{2} \cup K_{3}$, we define

$$
\begin{aligned}
\tilde{\Phi}_{\phi}(L)=\sum_{\rho}\left(t_{1}^{\sum_{\tau \in K_{1} \cap K_{2}} W_{\phi}(\tau, \rho)} t_{2}^{\sum_{\tau \in K_{2} \cap K_{3}} W_{\phi}(\tau, \rho)} t \sum_{3}^{\sum_{\tau \in K_{3} \cap K_{1}} W_{\phi}(\tau, \rho)}\right) \\
\in \mathbb{Z}\left[t_{1}, t_{2}, t_{3}\right] /\left(t_{1}^{4}=t_{2}^{4}=t_{3}^{4}=1\right),
\end{aligned}
$$

where $K_{i} \cap K_{j}$ denotes the set of crossing points between $K_{i}$ and $K_{j}$ and $\rho$ runs all proper colorings of the diagram by $T_{2}$.

Proposition 6.1 We have that $\widetilde{\Phi}_{\phi}(L)$ is invariant under Reidemeister moves.

Proof This follows from the fact that $\phi=\chi_{\left(a_{1}, a_{2}\right)}+\chi_{\left(a_{2}, a_{1}\right)} \in Z_{Q+}^{2}\left(T_{2} ; \mathbb{Z}_{4}\right)$.

Direct calculation shows that $\widetilde{\Phi}_{\phi}(\mathrm{BL})=2+2 t_{1}^{2} t_{2}^{2}+2 t_{2}^{2} t_{3}^{2}+2 t_{3}^{2} t_{1}^{2}$ and $\widetilde{\Phi}_{\phi}(\mathrm{TL})=8$, where $\mathrm{BL}$ denotes the Borromean rings and TL denotes the 3-component trivial link. Therefore $\widetilde{\Phi}_{\phi}(L)$ can be used to distinguish the Borromean rings from the trivial link. Further, since we are working with $T_{2}$, it follows that $\widetilde{\Phi}_{\phi}(L)$ is invariant under self-crossing changes. Hence the result above shows that the Borromean rings are not link-homotopic to the 3-component trivial link. Essentially speaking, the reason why $\widetilde{\Phi}_{\phi}(L)$ can tell the difference between the Borromean rings and the trivial link is that the Borromean rings are alternating. The writhe of a crossing between two components does not depend on the position of the third component, hence if the linking number of two components is zero then the third component has no effect on the quandle 2-cocycle invariant (associated with $T_{n}$ ). However the sign $\epsilon(\tau)$ we used here contains some information of the position of the third component. This is the reason why positive quandle 2-cocycle can be used to distinguish the Borromean rings and the trivial link. We remark that although for any quandle 2-cocycle of $T_{n}$ the state-sum invariant cannot distinguish the Borromean rings and the trivial link, A Inoue [18] used a 2-cocycle of a quasitrivial quandle to show that the Borromean rings are not link-homotopic to the 3-component trivial link. Note that the link-homotopy invariants defined by A Inoue in [18] have the same value on the Borromean rings and the 3-component trivial link if we work with the trivial quandles. 
The second example concerns the Fox 3-coloring. As we mentioned before, the diagram of knot 74 in Figure 12 contains a trivially colored crossing point if we consider the Fox 3-colorings. A natural question is which kind of knot diagram contains a trivially colored crossing point (associated with $R_{3}$ ). For example if the determinate of the knot is not divisible by 3 then there exists no nontrivial Fox 3 -coloring, hence each crossing point is a trivially colored crossing point. We end this paper by a simple sufficient condition to this question, which shows that the knot diagram in Figure 12 contains a trivially colored crossing point without needing to list all the proper colorings.

Proposition 6.2 Let $K$ be a knot diagram and consider the Fox 3-colorings. If $\sum_{\tau} \epsilon(\tau)$ is not divisible by 3 , then $K$ contains a trivially colored crossing point.

Proof Recall that $R_{3}=\{0,1,2\}$ with quandle operations $i * j=2 j-i(\bmod 3)$. Consider the coboundary

$$
\phi=\chi_{(0,1)}+\chi_{(1,0)}+\chi_{(1,2)}+\chi_{(2,1)}+\chi_{(2,0)}+\chi_{(0,2)} \in H_{Q+}^{2}\left(R_{3} ; \mathbb{Z}_{3}\right) .
$$

Since $\phi=\delta \chi_{0}$ it follows that $\Phi_{\phi}(K)=\sum_{\mathrm{Col}_{3}(K)} 0$ (here we write $\mathbb{Z}_{3}=\{0,1,2\}$ ). On the other hand, for each nontrivially colored crossing point $\tau$, the contribution of $\tau$ to $\Phi_{\phi}(K)$ is $\epsilon(\tau)$. Therefore if $K$ contains no trivially colored crossing points we have $\sum_{\tau} \epsilon(\tau)=0(\bmod 3)$. The result follows.

Acknowledgements We thank the referee for helpful comments. We also thank Kokoro Tanaka and Seiichi Kamada for pointing $[2 ; 22]$ to us respectively. The authors are supported by NSFC grant number 11171025. The first author is also supported by NSFC grant number 11301028 and the Fundamental Research Funds for Central Universities of China grant number 105-105580GK.

\section{References}

[1] J S Carter, A survey of quandle ideas, from: "Introductory lectures on knot theory", (L H Kauffman, S Lambropoulou, S Jablan, J H Przytycki, editors), Ser. Knots Everything 46, World Sci. Publ., Hackensack, NJ (2012) 22-53 MR2885229

[2] J S Carter, M Elhamdadi, M Saito, Twisted quandle homology theory and cocycle knot invariants, Algebr. Geom. Topol. 2 (2002) 95-135 MR1885217

[3] J S Carter, D Jelsovsky, S Kamada, L Langford, M Saito, Quandle cohomology and state-sum invariants of knotted curves and surfaces, Trans. Amer. Math. Soc. 355 (2003) 3947-3989 MR1990571

[4] J S Carter, D Jelsovsky, S Kamada, M Saito, Computations of quandle cocycle invariants of knotted curves and surfaces, Adv. Math. 157 (2001) 36-94 MR1808844 
[5] J S Carter, D Jelsovsky, S Kamada, M Saito, Quandle homology groups, their Betti numbers and virtual knots, J. Pure Appl. Algebra 157 (2001) 135-155 MR1812049

[6] J S Carter, M Saito, Canceling branch points on projections of surfaces in 4-space, Proc. Amer. Math. Soc. 116 (1992) 229-237 MR1126191

[7] J S Carter, M Saito, Knotted surfaces and their diagrams, Math. Surveys and Monographs 55, Amer. Math. Soc. (1998) MR1487374

[8] S Carter, S Kamada, M Saito, Surfaces in 4-space, Encyclopaedia of Math. Sciences 142, Springer, New York (2004) MR2060067

[9] W E Clark, M Elhamdadi, M Saito, T Yeatman, Quandle colorings of knots and applications, J. Knot Theory Ramifications 23 (2014) 1450035, 29 MR3253967

[10] M Eisermann, The number of knot group representations is not a Vassiliev invariant, Proc. Amer. Math. Soc. 128 (2000) 1555-1561 MR1657727

[11] R Fenn, C Rourke, Racks and links in codimension two, J. Knot Theory Ramifications 1 (1992) 343-406 MR1194995

[12] R Fenn, C Rourke, B Sanderson, Trunks and classifying spaces, Appl. Categ. Structures 3 (1995) 321-356 MR1364012

[13] R Fenn, C Rourke, B Sanderson, James bundles, Proc. London Math. Soc. 89 (2004) 217-240 MR2063665

[14] R H Fox, A quick trip through knot theory, from: "Topology of 3-manifolds and related topics", (M K Fort Jr, editor), Prentice-Hall, Englewood Cliffs, NJ (1962) 120-167 MR0140099

[15] C A Giller, Towards a classical knot theory for surfaces in $\mathbb{R}^{4}$, Illinois J. Math. 26 (1982) 591-631 MR674227

[16] F Harary, L H Kauffman, Knots and graphs, I: Arc graphs and colorings, Adv. in Appl. Math. 22 (1999) 312-337 MR1675756

[17] B Ho, S Nelson, Matrices and finite quandles, Homology Homotopy Appl. 7 (2005) 197-208 MR2175299

[18] A Inoue, Quasitriviality of quandles for link-homotopy, J. Knot Theory Ramifications 22 (2013) 1350026, 10 MR3070837

[19] A Inoue, Y Kabaya, Quandle homology and complex volume, Geom. Dedicata 171 (2014) 265-292 MR3226796

[20] D Joyce, A classifying invariant of knots, the knot quandle, J. Pure Appl. Algebra 23 (1982) 37-65 MR638121

[21] S Kamada, Knot invariants derived from quandles and racks, from: "Invariants of knots and 3-manifolds”, Geom. Topol. Monogr. 4 (2002) 103-117 MR2002606

[22] S Kamada, V Lebed, K Tanaka, The shadow nature of positive and twisted quandle cocycle invariants of knots (2014) arXiv:1409.4072v2

[23] R A Litherland, S Nelson, The Betti numbers of some finite racks, J. Pure Appl. Algebra 178 (2003) 187-202 MR1952425

[24] T W Mattman, P Solis, A proof of the Kauffman-Harary conjecture, Algebr. Geom. Topol. 9 (2009) 2027-2039 MR2550465 
[25] S V Matveev, Distributive groupoids in knot theory, Mat. Sb. 119(161) (1982) 78-88, 160 MR672410 In Russian; translated in Math. USSR-Sb. 47 (1984) 73-83

[26] T Mochizuki, Some calculations of cohomology groups of finite Alexander quandles, J. Pure Appl. Algebra 179 (2003) 287-330 MR1960136

[27] M Niebrzydowski, J H Przytycki, Homology of dihedral quandles, J. Pure Appl. Algebra 213 (2009) 742-755 MR2494367

[28] M Polyak, Minimal generating sets of Reidemeister moves, Quantum Topol. 1 (2010) 399-411 MR2733246

[29] J H Przytycki, 3-coloring and other elementary invariants of knots, from: "Knot theory”, (V F R Jones, J Kania-Bartoszyńska, J H Przytycki, V G Traczyk, Pawełand Turaev, editors), Banach Center Publ. 42, Polish Acad. Sci., Warsaw (1998) 275-295 MR1634462

[30] J H Przytycki, Distributivity versus associativity in the homology theory of algebraic structures, Demonstratio Math. 44 (2011) 823-869 MR2906433

[31] J H Przytycki, A S Sikora, Distributive products and their homology, Comm. Algebra 42 (2014) 1258-1269 MR3169627

[32] D Rolfsen, Knots and links, Math. Lecture Series 7, Publish or Perish (1976) MR0515288

[33] D Roseman, Reidemeister-type moves for surfaces in four-dimensional space, from: "Knot theory", (VFR Jones, J Kania-Bartoszyńska, J H Przytycki, V G Traczyk, Pawełand Turaev, editors), Banach Center Publ. 42, Polish Acad. Sci. (1998) 347380 MR1634466

[34] C Rourke, B Sanderson, There are two 2-twist-spun trefoils (2002) arXiv: math.GT/0006062

[35] S Satoh, A Shima, The 2-twist-spun trefoil has the triple point number four, Trans. Amer. Math. Soc. 356 (2004) 1007-1024 MR1984465

[36] M Takasaki, Abstraction of symmetric transformations, Tôhoku Math. J. 49 (1943) 145-207 MR0021002

[37] L Vendramin, On the classification of quandles of low order, J. Knot Theory Ramifications 21 (2012) MR2926571

School of Mathematical Sciences, Beijing Normal University

Laboratory of Mathematics and Complex Systems

Ministry of Education, Beijing 100875, China

czy@bnu.edu.cn, hzgao@bnu.edu.cn

Received: 16 April $2014 \quad$ Revised: 18 September 2014 
